ROCZNIKI KULTUROZNAWCZE

Tom XII, numer $4-2021$

DOI: http://doi.org/10.18290/rkult21124-4

ORLANDO TODISCO OFMConv

\title{
RECUPERO DELLA FIGURA DEL PADRE AI MARGINI DEL MEDIOEVO FRANCESCANO
}

L'operazione dell'età moderna è pensabile come una colossale messa in crisi di tutto ciò che non filtra attraverso la ragione critica, oggettivante e universalizzante, padrona del territorio. Questo capitolo è da ricondurre all'indole dell'essere come diritto, all'illimitatezza dell'agire e dunque alla rivendicazione di totale autonomia, di cui la ragione è voce intransigente. Ora, volendo tentare il cambio di rotta o proporre un altro possibile percorso, vorremmo attingere al fondo del medioevo francescano alcune suggestioni filosofico-teologiche in vista di una diversa lettura del reale e dunque disporre di un altro registro, costituito non dall'essere-come-diritto e dunque non dal primato della ragione, ferma al "che cosa" di una cosa, ma dall'essere come-dono e dunque dal primato della libertà creativa di segno oblativo, anima del sentire francescano. In fondo si tratta dell'essere-come-dovere, come obbligo, come gratitudine, come riconoscimento dell'altro, conseguente alla coscienza che si è in quanto voluti da chi poteva non volerci. In siffatto contesto pare non possa non aver luogo la riscoperta della figura del "padre", che è appunto il modello della libertà creativa di segno oblativo, o anche la cifra dell'essere-come-dono. Il che ha luogo trascendendo la verità con la bontà attraverso l'essere come dono, che a sua volta rimanda alla libertà creativa di segno oblativo del donatore, il che in ultimo rinvia a un'ontologia dell'essere pluridimensionale e cioè per un verso oggetto della ragione o versante fenomenico e per un altro trascendente il versante razionale grazie alla libertà creativa di cui conserva il segreto. E' alla luce di tale ontologia che è possibile attingere al fondo

Prof. ORlando Todisco, Pontificia Facoltà Teologica San Bonaventura - Seraphicum; indirizzo postale: Collegio Internazionale Seraphicum, Via del Serafico 1, 00142 Roma, Italia; e-mail: orlando.todisco@virgilio.it. 
abissale del Medioevo francescano rivalutando la figura del padre, segnato non dallo spirito autoritario e possessivo, ma da uno stile di servizio e di sostegno nel contesto di una sorta di affratellamento cosmico.

\section{INTRODUZIONE}

Due le prospettive entro cui la storia si muove da sempre, la prospettiva del dominio e la prospettiva del servizio, o anche la volontà di potenza e la volontà di libertà $\mathrm{o}$, più radicalmente, l'essere come affermazione e l'essere come donazione. E' l'andamento pendolare della storia. La linea che finora si è imposta è costituita dalla volontà di potenza nel contesto dell'essere come diritto-a-essere, fonte di rivendicazione. Se questa è la linea vincente, la proposta francescana rovescia l'impianto, passando dall'autoaffermazione all'autodonazione dell'essere, o anche, e in forma abbreviata, passando dall'essere come diritto-a-essere all'essere come dono-di-essere. Siamo oltre l'orizzonte greco-romano come oltre l'orizzonte sia moderno che contemporaneo, che escludono l'atto creativo divino come atto sorgivo del carattere oblativo dell'essere. Ebbene, ciò che ci proponiamo è di mostrare che il volontarismo francescano, che riconosce il primato all'ens volitum, nel senso che l'essere non è neutro - l'essere in quanto essere - ma voluto, progettato, amato, è compendiabile nella figura del "padre", essenzialmente "creativa" e "oblativa". Per misurarne l'originalità è forse utile tenere sullo sfondo la prospettiva moderna e contemporanea, ispirata all'essere essenzialmente neutro, appannaggio di chi per primo vi pianta la bandiera, compendiabile nella figura del figlio che ripudia il padre e la sua visione dell'essere - l'età medievale -, non perché autoritaria, ma perché oblativa, e cioè gratuita, senza perché, e dunque fondata non sulla ragione, ma sul potere sacro della tradizione e sull'autorità del proponente. La volontà oblativa, che per il francescano fa tutt'uno con la figura del padre, emerge forse con più forza se rapportata alla volontà possessiva tipica dell'uomo moderno, impegnato a diventare "maître et posseseur du monde" (Descartes). E' la donazione gratuita della vita e di quanto questa comporta per la sua crescita il tratto distintivo della figura francescana del padre, immagine meno inadeguata di Dio, elargitore della vita e con essa di ogni bene, esemplare supremo per la corretta configurazione del nostro stile di vita ${ }^{1}$. Dunque, nucleo centrale della

\footnotetext{
${ }^{1}$ Bonaventura, II Sent., d. 25, p. I, q. 5: «Item, aeque essentiale est animae habere libertatem arbitrii, sicut esse ad imaginem Dei»; ibid., d. 25, p. 1, dub. 1: «Cum anima sit imago Dei secundum
} 
paternità è una sorta di strutturale attenzione per l'altro - famiglia, comunità, nazione - non inquinata dal peso dell'io concupiscente, grazie alla forza purificatrice della povertà, tesa ad ampliare gli spazi, a elevare la qualità del percorso, al fine di ritrovare il senso dell'essere. E' possibile compendiare tutto ciò nell'espressione scotista sulle labbra del padre: "amo: volo ut sis" ti amo e cioè voglio che tu esista per davvero ${ }^{2}$.

\section{ALLA FONTE DELLA DIMENSIONE OBLATIVA DELL'ESSERE}

La forza argomentativa a sostegno della dimensione oblativa della figura del padre è tratta anzitutto dalla tesi della "creazione ex nihilo", quale gesto altamente significativo della paternità divina, non da intendere come esaltazione della sua potenza creatrice, ma come effusione del dono della vita in totale gratuità - ex nihilo. E' la versione francescana della paternità, secondo cui il mondo e noi in esso siamo dono della volontà divina, sollecitati ad attivare una condotta analoga, innovativa e oblativa ${ }^{3}$. L'assenza dal quadro culturale generale del pensare sia greco-romano che moderno-contemporaneo della

liberum arbitrium, ut dicit Richardus». Significativa la definizione del libero arbitrio come potere della volontà di realizzare il bene: «Liberum arbitrium 'est potestas servandi rectitudinem', sicut dicit Anselmus; sed maior est potestas servandi rectitudinem in bonis quam in malis, in confirmatis quam in non confirmatis: ergo liberum arbitrium contingit secundum magis et minus participari» (II Sent., d. 25, p. II, q. 1). Interessante la sottolineatura che la libertà conserva la sua dignità d'eccellenza (eccellentiae dignitas) se in grado di realizzare il bene senza alcuna coazione: «Libertas arbitrii est quaedam facultas sive potestas servandi rectitudinem. Haec autem facultas, prout privationi coactionis coniuncta est, habet quandam dignitatem: dignitatis enim est et potestatis non posse cogi. Et sic in liberi arbitrii intellectu tria clauduntur, videlicet a coactione immunitas, excellentiae dignitas et potestas sive facultas» (ibid., q. 1, resp.).

${ }^{2}$ Hanna ARENDT, La vita della mente, trad. Giorgio Zanetti (Bologna: Il Mulino, 1987), 458: «L'io che vuole, allorché nella sua manifestazione suprema afferma «Amo: volo ut sis», «Io ti amo: voglio che tu sia» - e non «voglio averti» o «voglio dominarti» - si dimostra capace del medesimo amore con cui Dio ama gli uomini, che Egli creò solo perché volle la loro esistenza, che Egli ama senza desiderarli». Per misurare la distanza di questa prospettiva da quella greco-romana si rifletta su ciò che il giurista Gaio del II secolo d.C. scrive: "Non esistono altri uomini che abbiano sui propri figli un potere come quello che noi abbiamo sui nostri". Cf. il commento di Eva CANTARELLA, Come uccidere il padre. Genitori e figli da Roma a oggi (Milano: Feltrinelli, 2020), 17. E' estremamente significativo quanto l'autrice richiama e cioè che, a partire dai Sette re di Roma, a metà dell'VIII secolo a. C., fino al VI secolo d. C. il potere di vita e di morte dei padri sui figli è assoluto, e, come

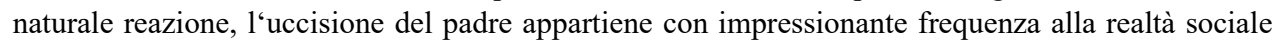
di ogni famiglia romana.

${ }^{3}$ BonAVENTURA, II Sent., d. 25, p. I, dub. 3: «Et hoc modo reperitur in creatura et Deo conformiter et secundum eandem rationem, non conformitate et unitate univocationis, sed potius analogiae et consimilis habitudinis, quae inter Deum et creaturam esse potest». 
"creatio ex nihilo" va messa in grande evidenza, perché significativa dell'indole auto-affermativa dell'essere, con una sua storia, che però non fa capo ad alcun agente esterno, frutto piuttosto della dinamica degli elementi che lo compongono.

\section{A. LA PLURALITÀ DELLE FONTI ARGOMENTATIVE}

Il tema della "creatio ex nihilo" è riassuntivo di una serie di capitoli filosofici attraverso i quali è possibile misurare la distanza teoretica della prospettiva francescana da quella sia greco-romana che moderno-contemporanea, per l'una qualificante, per le altre estranea, anzi irrilevante. Da qui la contrapposizione tra le due visioni, di cui occorre cogliere la forza teoretica, in vista di una auspicata rigenerazione culturale. Il filo rosso della ricerca è che nell'ottica francescana l'oblatività - la dimensione paterna - esprime l'anima dinamica dell'essere - Dio è in quanto si dona («ideo est quia donum») ${ }^{4}$, nell'eterno in seno alla Trinità, nel tempo attraverso la creazione e la redenzione; la natura è in quanto manifesta progressivamente le sue molte energie attraverso l'immenso panorama dei fenomeni terrestri e celesti; l'uomo è in quanto chiama all'essere altre creature e trasforma il giardino, che gli è stato affidato, in un luogo luminoso e accogliente; la ricerca è coinvolgente se e in quanto apre nuove prospettive. L'essere è il suo darsi. Il che significa che il divenire, attraverso cui l'essere si manifesta, non è da intendere come ricerca di ciò che non si ha o non si è, ma come donazione di ciò che si è e si ha, attraverso rinnovate trame di relazioni ${ }^{5}$, o anche come desiderio non di ciò che manca ma di ciò che già si possiede, da esplorare con rinnovata passione. La consapevolezza di ciò che si è e si ha, da approfondire sempre di nuovo, è funzionale alla dimensione oblativa, che esprime al meglio l'ontologia francescana, per la quale il verbo 'essere' fa tutt'uno con il verbo 'amare', che è al tempo stesso un verbo attivo - rappresenta uno scambio - e un verbo transitivo - indica il passaggio del dono. Il nucleo oblativo della 'creazione' risulta corroborato dal 'nihil' del sintagma "creatio ex nihilo", cifra della

${ }^{4}$ Bonaventura, I Sent., d. 18, dub. 1.

${ }^{5}$ Gianni Vattimo, La vita dell'altro. Bioetica senza metafisica (Napoli: Marco Constantino, 2006), 172: “'Tutto è grazia' (Bernanos). In termini filosofici diremmo con Heidegger che l'essere, se è qualcosa, è evento, Ereignis. Certo, es gibt Sein: ma solo nel senso che es, das Sein, gibt. L'essere dona, questa è la sua sola pensabile 'essenza'. Non propongo di saltare semplicemente fuori dal linguaggio filosofico per passare a quello della rivelazione cristiana. Ciò che intendo dire è che la stessa filosofia - almeno nella forma che mi appare la più capace di corrispondere alla nostra epoca e alla nostra specifica vocazione storica - deve ricorrere al messaggio cristiano per risolvere le contraddizioni e aporie della metafisica». 
radicale gratuità, dal momento che la creatura, essendo "ex se" un niente ex nihilo - è gratuita, e cioè non è perché vantasse un qualche diritto.

Infine, un'altra fonte di questa dimensione oblativa della libertà creativa è costituita dal "sentire" di Francesco, ispirato non più al padre terreno che impone la sua volontà, ma al padre che è nei cieli, fonte primaria di ogni paternità, secondo l'Apostolo per il quale ogni paternità sulla terra viene dalla paternità del cielo $(E f 3,15)$. E' nell'orizzonte onnicomprensivo di tale paternità la distanza tra Francesco e il monachesimo della separazione dal mondo, la cui indole si manifesta a condizione che ci si collochi al di là di ogni antropocentrismo, in nome di quella fraternità che coinvolge tutte le relazioni, comprese quelle con gli elementi naturali, con gli animali, finanche con la morte. "Ponendosi come programma un ideale positivo, aperto all'amore per tutte le creature e tutta la creazione, ancorato alla gioia e non più alla tetra accidia o alla tristezza, rifiutando di essere il monaco ideale della tradizione votata al pianto", egli ridà smalto e vigore alla figura paterna, liberandola da quella forma di opacità che fa tutt'uno con l'identificazione con un percorso specifico e con la presa di distanza da tutti gli altri. "Schiudendo alla spiritualità cristiana la cultura laica cavalleresca dei trovatori e la cultura laica popolare del folclore paesano con i suoi animali, il suo universo naturale, il meraviglioso francescano ha infranto le chiusure che la cultura clericale aveva imposto alla cultura tradizionale ${ }^{6}$ ". E' questa accoglienza del reale nella pluralità delle sue espressioni la traduzione della paternità francescana, festosa e oblativa a un tempo.

Dall'insieme risulta che sia l'età antica che l'età moderna-contemporanea, per le quali la creazione gratuita dell'essere è estranea o comunque non qualificante, la dimensione oblativa rimane secondaria o piuttosto festiva è più un'eccezione che un modo d'essere e di pensare - subordinata alla dimensione potestativa e dominatoria, nel senso che si risolve nel dono, funzionale al contro-dono secondo la logica del "do ut des". La figura del padre in senso francescano, cifra di colui che dona in radicale gratuità, risulta del tutto assente, sopraffatta dalla figura del figlio che nell'età moderna rivendica con violenza un ruolo potestativo. Il suo venire nella non-latenza o il suo apparire nel tempo comporta la contestazione radicale del padre e di quanto questo significa - è il carattere metodico del dubbio cartesiano.

\footnotetext{
${ }^{6}$ Jacques Le Goff, San Francesco d'Assisi, trad. Lisa Baruffi e Amedeo De Vincentiis (Bari: Laterza, 2002), 72.
} 


\section{B. IN PRINCIPIO LA VIOLENZA?}

Pare pacifico il rilevare dal punto di vista storico che l'uomo risulti violento, oltre che nel suo operare, nel suo stesso essere. Heidegger ritiene che la vera definizione dell'uomo non è quella piuttosto "zoologica" di "animale razionale", ma quella che lo distingue da tutti gli altri viventi, e cioè la violenza. Questa gli è intrinseca, gli appartiene. Da una parte si ha l'essente nella sua totalità, e dall'altro l'uomo violento che "a ragione del suo agire violento $\mathrm{e}$ in quanto situato in esso usa la violenza nei confronti del predominante" 7 . La violenza non è un tratto soltanto umano. Essa appartiene alle stesse potenze naturali. "Il far violenza del dire poetico, del progetto del pensiero, del formare costruente, dell'azione creatrice di stati, non è l'esercitarsi di poteri che l'uomo possiede in proprio, ma un dominare e accordare le potenze grazie alle quali l'essente si schiude, come tale, per via dell'uomo che entra in lui. Questo aprirsi dell'essente rappresenta la violenza che l'uomo deve padroneggiare onde essere, in questo far violenza in mezzo all'essere, se stesso, vale a dire se stesso come essere storico" ${ }^{8}$. E' il modo occidentale di intendere l'uomo e di osservarne la presenza nel mondo, segnati a diverso titolo da quell'attività produttiva entro cui si raccoglie e prende forma la violenza. L'orizzonte che si dispiega comprende tutti gli enti, giustificandone immanentisticamente il darsi per il tempo concesso dalla physis, messa a frutto dall'uomo. Essere e diritto a-essere sono un tutt'uno.

Leggendo la storia dell'Occidente nel quadro dell'onto-teologia, ovvero alla luce della riduzione dell'essere a ente, manipolabile e calcolabile, Heidegger interpreta l'azione creativa divina come il sigillo della violenza sulle cose, grazie a cui queste vengono all'essere imponendosi. Lo scenario che si dischiude è quello dell'effettività o della produzione realizzata dall'attività causale del sommo ente. Viene ricondotta a questa fondamentale relazione causale la parentela e dunque il naturale confluire della filosofia antica nella prospettiva teologica medievale. "La creazione - scrive Heidegger - è interpretata richiamandosi in qualche modo alla produzione. L'ontologia antica, nei suoi fondamenti e nei suoi concetti base, nonostante la sua differente origine, era, per così dire, tagliata su misura per la concezione cristiana e per il suo modo di concepire l'ente come ens creatum"". Dio è considerato come causa efficiente e l'ente creaturale come ciò che è accessibile, rappresentabile,

\footnotetext{
${ }^{7}$ Martin HeIDEgGer, Introduzione alla metafisica (Milano: Mursia, 1986), 158.

${ }^{8}$ Ibid., 164-65.

${ }^{9}$ Martin HeIDEGGer, I problemi fondamentali della fenomenologia (Genova: Il Melangolo, 1988), 113.
} 
a portata di mano, secondo lo stile del pensare successivo a quello grecomedievale, in sostanziale continuità. L'accento è posto sul fatto che l'ente risulta creato e cioè prodotto o anche causato. Il cristianesimo confermerebbe dunque l'origine del carattere violento dell'ente, legittimando le pretese di dominio. Ciò che interessa Heidegger è fondamentalmente "l'idea di creazione come riduzione 'violenta' della manifestatività dell'essere ad una dinamica precostituita, e puramente infra-ontica, di causa ed effetto" ${ }^{10}$. Non si trascuri l'indole della traiettoria lungo la quale il filosofo ripensa il testo rivelato: "La metafisica moderna da Descartes a Kant, ed oltre Kant anche la metafisica dell'idealismo tedesco, non è pensabile senza le concezioni fondamentali del cristianesimo. [...] è naturale che si spieghi la metafisica moderna nel suo complesso in base ai suoi due momenti essenziali: la concezione cristiana dell'ente come ens creatum e il fondamentale 'carattere matematico', riferendo il primo momento al contenuto e il secondo alla forma" "11. L'idea, dunque, di creazione ex nihilo sarebbe, a parere di Heidegger, il paradigma di un'azione violenta che rende la cosa creata disponibile sia come causa che come effetto.

\section{LA SCELTA STORIOGRAFICA FRANCESCANA}

Ora, il francescano non mette in discussione che nel contesto dell'autoaffermazione si sia imposta, e si imponga, l'esigenza a essere, provocando l'inevitabile scontro con l'altro che, alla luce delle proprie possibilità, rimuove o aggira qualunque ostacolo si frapponga alla loro realizzazione. E' la descrizione di ciò che è accaduto e accade. Ciò che il francescano mette decisamente in questione è che tutto ciò esprima il volto originario dell'essere, le sue istanze più proprie - il che comporterebbe la legittimazione del "Dio ingannatore", il cui atto creatore sarebbe all'origine dell'avventura della violenza $^{12}$. No, il primo atto del Dio creatore è segnato dalla benevolenza. Ed è sullo sfondo di questa presupposta visione di Dio che il peso della storia e il suo volto conflittuale vengono fatti ricadere sull'uomo, sul quale

\footnotetext{
${ }^{10}$ Giuseppina Strummiello, Il logos violato. La violenza nella filosofia (Bari: Dedalo, 1991), 252.

${ }^{11}$ Martin HeIDEgGer, La questione della cosa. La dottrina kantiana dei principi trascendentali (Napoli: Guida, 1989), 134.

${ }^{12}$ Dio ingannatore e genio maligno. Nota in margine alle Meditationes di Descartes, in Tullio GrEgORY, Mundana sapientia. Forme di conoscenza nella cultura medievale (Roma: Edizioni di Storia e Letteratura, 1992), 401-40. Il che non significa che Heidegger condivida l'ipotesi del 'Dio ingannatore'. Il suo obiettivo è di contestare l'ontologia tradizionale secondo cui Dio è un ente sia pure infinito. Pur condividendo la critica all'onto-teologia tradizionale, qui si cerca di mettere in luce quella che riteniamo il 'pomo della discordia' tra l'età medievale nella versione francescana e l'età moderna, costituito dalla concezione dell'essere, per l'una di segno oblativo, per l'altra rivendicativo.
} 
grava la responsabilità della piega della nostra avventura nel tempo ${ }^{13}$. Al seguito della parola divina, il disegno del francescano si fonda su uno specifico impianto valoriale, che non ruota più intorno all'autoaffermazione dell'essente dominante, animato dalla violenza, ma intorno all'essere come fonte di oblazione e dunque originariamente "pacifico". Non che si ignori il darsi della violenza come anima sotterranea della storia. Il francescano ritiene che non sia legittimo subordinare a questa logica di violenza spartiti teoretici che invece sono stati elaborati con altri intenti e sono stati intesi e proposti per generare un ben altro stile di vita. Il che vien detto in linea con la Rivelazione biblico-cristiana, a cominciare dall'atto creativo, alla cui luce siamo invitati a prendere coscienza di essere una risposta a una voce che ci chiama all'essere da un altrove che trascende l'ambito della natura e la sua legalità, grazie a un'onda oblativa entro cui vivere l'abbraccio cosmico ${ }^{14}$. Non si tratta di misconoscere la realtà, segnata dalla violenza, ma di proporre un altro modo di pensarla e di leggerla, non riportando all'origine del tempo ciò che è accaduto nel corso del tempo, ma recuperando l'intenzione di Colui che ha voluto ciò che avrebbe potuto non volere. L'uomo si trova nella situazione di decaduto - la colpa non è tanto un'intenzione maligna, quanto un consapevole modo d'essere. Tuttavia questo modo d'essere non è il suo essere. "E se lo è lo è nella forma della negazione. O del suo essere in colpa. 'Tu non sei quello!', è l'originaria voce della coscienza, che è coscienza di non essere prima ancora che di essere. Non essere quel che in realtà uno è: negligente, apatico, sempre un passo indietro rispetto alla costruzione del mondo" 15 . Nonostante l'onda del male, il francescano si lascia investire dalla luce calda dell'inizio, dall'irruzione del dono dell'essere, in un contesto di fiducia da parte del padrone che «prima di partire per un lungo viaggio dà la propria casa e tutto il suo potere ai suoi servi, invitandoli a essere vigili e creativi» (Mc 13,33-37).

${ }^{13}$ Itinerarium mentis in Deum (= Itin.), I, n. 7: «Sed advertens se a vero lumine ad commutabile bonum, incurvatus est ipse per culpam propriam, et totum genus suum per originale peccatum, quod dupliciter infecit naturam humanam, scilicet ignorantia mentem et concupiscentia carnem; ita quod excaecatus homo et incurvatus in tenebris sedet et coeli lumen non videt, nisi sucurrat gratia cum iustitia contra concupiscentiam, et scientia cum sapientia contra ignorantiam».

${ }^{14}$ E' significativa la risposta di Francesco al card. Ugolino che cercava di impedire che Francesco si allontanasse dall'Italia, diretto verso la terra degli infedeli rilevando che il suo movimento aveva bisogno di una guida e che nella curia vi erano molti nemici. Siamo a Firenze nel 1217. Contrariato dall'invito autorevole, Francesco replicò: «Credete o pensate, signore, che Dio abbia invitato i frati soltanto per queste regioni? Vi dico in verità che Dio ha scelto e inviato i frati per il vantaggio spirituale e la salvezza delle anime di tutti gli uomini del mondo intero» (Scripta Leonis, Rufini et Angeli Sociorum S. Francisci, cur. Rosalind B. Broke (Oxford: Clarendon Press, 1970), cap. 82, pp. 232-30, cit. da Chiara Frugoni, Un presepio con molte sorprese. San Francesco e il Natale di Greccio (Roma: Mauvais Livres, 2020), p.116, nota 52.

${ }^{15}$ Sergio Givone, Metafisica della peste. Colpa e destino (Torino: Einaudi, 2012 ), 64. 


\section{IN PRINCIPIO LA DIMENSIONE EFFUSIVA DELLA LIBERTÀ CREATIVA}

E' pacifica l'estraneità della prospettiva greco-pagana alla prospettiva biblico-cristiana, confermata dall'assenza dell'atto creativo originario, con il quale Dio ci chiama all'essere e subordina il mondo inferiore al nostro essere. Il francescano ritiene riduttiva la lettura della realtà in termini di "ens creatum" e cioè come effetto, disponibile e calcolabile ${ }^{16}$. Il contesto è un altro e ha un'altra tonalità. L'uomo deve guardare alla natura come alla sua casa ${ }^{17}$ e alle creature inferiori come ai suoi inquilini ${ }^{18}$. Non c'è spazio per la violenza. Al primo posto non c'è l'enigma dell'essere, né la falce della morte. In principio la libertà oblativa del creatore, che creando manifesta la sua benevolenza ${ }^{19}$. L'orizzonte non è segnato originariamente dal negativo da emarginare, né dal bisogno da soddisfare, né dalla necessità di colmare il vuoto; né il compito primario, che incombe su di noi, è di contrastare l'onda devastante della violenza sopraffattrice. L'essere non ha il volto della violenza, né l'esistenza è segnata dal dolore. Il thauma o sorpresa, con cui Platone e Aristotele dicono che sia sorta la filosofia, non è costituito dal turbamento provocato dal dolore e dalla morte, o anche dall'enigmaticità della physis. Come, allora, guardare all'origine della nostra avventura? Nella versione biblico-cristiana

${ }^{16}$ Bonaventura, II Sent., d. I, p. II, a. 2, q. 2: «Aliquid, propter quod res est, aut est ad supplendum indigentiam, sicut domus propter hominem, et calceus propter pedem; aut ad suscipiendum eius influentiam, sicut homo propter Deum. Quod ultimo modo habet rationem finis, nobilius est, quod primo modo non. Quia enim homo omnibus indiget, Angelus nullo nisi Deo; ideo omnia propter hominem, non omnia propter angelum».

${ }^{17}$ La creazione è la "casa" costruita da Dio per l'uomo: «Haec igitur sensibilis corporalium machina est tamquam quaedam domus a summo opifice homini fabricata, donec ad domum veniat non manufactam in caelis» (Breviloquium (= Brev.) II, cap. 4, n. 5). Tutto è funzionale alle necessità dell'uomo ('ad supplemendum indigentiam'). L'uomo ha bisogno di tutto, dunque «omnia propter hominem» (II Sent., dist. 1, p. 2, q. 2). Questa casa è stata creata nel tempo dal nulla, sicché «excluditur error manicheorum ponentium pluralitatem principiorum» (Brev. II, cap. 1, n. 2). Da qui la conclusione che l'uomo può abbellirla servendosi di tutto il materiale possibile, purché non la trasformi in caverna, diventandone prigioniero, e non trascuri il compito primario di arricchirla con relazioni di universale fraternità.

${ }^{18}$ II Sent., d. XIX, a. 1, q. 2: «creaturae, quae solum tenent rationem vestigii, factae sunt propter creatram rationalem, scilicet hominem».

${ }^{19}$ Scotus, Ordinatio (= Ord.) I, d. 2, p. 1, q. 1-2, n. 83: «Philosophus antecedens concessit, scilicet quod aliquid contingenter causatur et negavit consequens, intelligendo de velle Dei, scilicet quod Prima Causa contingenter causat»". Ord. I, d. 38, q. u., n. 9: «Intellectus divinus ex necessitate naturae est speculativus [...] Quando igitur arguis si voluntas non possit non velle intellectum speculari, ergo non est summe libera, non sequitur, quia libertas eius non est ad intrinseca (quae quasi praecedunt actum eius), sed libertas eius est ad omnia factibilia, et ideo necesse est ponere primam dceterminaionem in voluntate respectu factibilium; non sic autem esset si intellectus voluntatem praedeterminaret». 
la meraviglia (thauma), che sveglia il pensare, è costituita dall'incomparabile bellezza di ciò che ci circonda, di ciò che siamo, del traguardo che ci attende ${ }^{20}$. Il tutto è da ricondurre a Colui la cui essenza è costituita dall'amore effusivo ${ }^{21}$; o anche, alle inenarrabili ricchezze che si impongono guardando il cielo dentro di noi, sopra di noi, intorno a noi. L'atto creativo divino è fonte inesauribile di tesori ${ }^{22}$. Occorre risvegliare il senso del bello, del buono, del giusto, che ci abita, al punto che, anche se incapaci di definirlo, sappiamo cosa comporti il fare una cosa bella, vera, buona, giusta. Il fondo dell'anima è animato da quanto di nobile è possibile ipotizzare. La teologia sviluppa questa prospettiva portandola al di là di ogni immaginazione allorché afferma che siamo a immagine stessa di Dio. Al vertice di tutto l'Uomo-Dio, esemplare di tutto ciò che viene chiamato all'essere. E' il progetto che si fatica a condividere per la sua luminosa bellezza. Non si tratta di una prospettiva utopica, ma di una realtà da esplorare, entro cui siamo e viviamo, per lo più inconsapevolmente. E' l'opera di Dio. Non ne siamo noi gli autori. Ne siamo i beneficiari, che per una serie di eventi, con cui l'abbiamo messa ai margini della nostra storia, abbiamo consentito che fosse avvolta da una deprecabile oscurità, avvertendone però il richiamo e sentendone la nostalgia ${ }^{23}$. Dunque, si tratta di metter mano a un'altra costruzione e di pensare in un ben diverso orizzonte valoriale, alla cui luce rileggere il presente e stimolare l'affermazione di un'altra sensibilità.

${ }^{20}$ Bonaventura, II Sent, d. XV, a. 2, q. 1: «Insinuat etiam Scriptura multo excellentius, cum dicit: Faciamus hominem ad imaginem et similitudinem nostram, praesit piscibus maris etc. Quia enim homo rationis capax est, ideo habet libertatem arbitrii et natus est piscibus dominari; quia vero per similitudinem natus est in Deum immediate tendere, ideo omnes creaturae irrationales ad ipsum ordinantur, ut mediante ipso in finem ultimum perducantur».

${ }^{21}$ Scotus, Ord. I, d. 10, q. u., n. 9: «In Deo [...] est formaliter voluntas ex natura rei et hoc sub ratione principii productivi liberi respectu amoris, et patet quod est ibi sine imperfectione; ergo erit in Deo principium producendi amorem, et hoc secundum proportionem perfectionis suae, ita quod sicut voluntas creata est principium producendi amorem tantum quanto amore est ipsa potest amare obiectum (qui dicitur amor adaequatus), ita ista voluntas est principium producendi amorem tantum quanto ipsa nata est amare obiectum infinitum, - nihil autem est infinitum nisi sit ipsa essentia divina, igitur ille amor est essentia divina».

${ }^{22}$ Thomas Merton, Gli abissi infiniti del cielo, trad. Chiara Benedetti (Brescia: Queriniana, 2019).

${ }^{23}$ Itin. III, n. 4: «Desiderium autem prinicipaliter est illius quod maxime ipsum movet. Maxime autem movet quod maxime amatur; maxime amatur esse beatum; beatum autem esse non habetur nisi per optimum et finem ultimum: nihil igitur appetit humanum desiderium nisi quia summum bonum, vel quia est ad illud, vel quia habet aliquam effigien eius». 


\section{LA LUCE DEL PADRE}

\section{A. IL PADRE ALL'ORIGINE DI TUTTO}

Lo spazio che il francescano riserva al "padre" è sacro e inviolabile, perché riguarda l'origine, la genesi, il principio di tutto ciò che è, umano e divino, eterno e temporale. Aprendo il discorso sul mistero della Trinità divina, si ha anzitutto il "Padre", principio dei principi, fonte originaria di tutti i possibili rivoli di vita ${ }^{24}$. E' il punto d'avvio che dà il tono a tutto il seguito e dunque da preservare con cura. Da qui l'attenzione di Bonaventura per la figura del Padre nel mistero della Trinità, ben consapevole di misurarsi con la fonte dell'essere, dunque da esplorare con rigore in vista della luce che inevitabilmente riversa sull'intero scenario dell'essere, nell'eterno e nel tempo ${ }^{25}$. Egli dedica il solo articolo dell'ottava questione circa il Mistero della Trinità al tema della "primitas" del Padre, primo principio. Descrivendone le proprietà è possibile ammirare l'immenso panorama che si viene dischiudendo. «Per il fatto di essere primo, è perfettissimo nel produrre, sommamente capace di originare nell'emanare e fecondissimo nel diffondere [...] Da ciò segue che il primo Principio, poiché è primo, è eterno con la pienezza di tutti gli attributi eccellenti. Perciò in esso stanno tutte le cose e tutti gli atti di comprensione» ${ }^{26}$. Il duplice aspetto del Padre è che per un verso è "innascibile" nel senso che non ha origine e di conseguenza che è assolutamente primo - anteriore a ogni processione -, e per l'altro è genitore, nel senso che è la proprietà generativa a qualificarlo - è tale proprietà a fare del padre il padre. La generazione precede e giustifica la nozione stessa di padre. Perché genera, Dio è padre, non viceversa, come sosteneva Tommaso per il quale la generazione è del padre in quanto ha la capacità di generare, non presupponendo la quale non si dà spiegazione esatta dell'atto generativo - prima dell'atto, colui che agisce

${ }^{24}$ Brev. Prol. n. 5: «Inchoandum est ab exordio, hoc est, ut cum mera fide ad Patrem luminum (Gc 1,17) accedamus, flectendo genua cordis nostri, ut ipsed per Filium uum in Spiritu Sancto det nobis veram notiam Iesu Christi et cum notitia amorem eius [...] et per hanc notitiam pervenire ad plenissimam notitiam ex excessivum amorem beatissimae Trinitatis».

${ }^{25}$ De Mysterio Trinitatis VIII, ad ob. 7: «Quia enim Pater producit Filium et per Filium et cum Filio producit Spiritum Sanctum; Ideo Deus Pater per Filium cum Spiritu Sancto est principium omnium creatorum; nisi enim eos. produceret ab aeterno, non per illos producere posset ex tempore; et ideo ratione illius productionis in Trinitate recte dicitur esse fons vitae».

${ }^{26}$ De Mysterio Trinitatis VIII, concl. resp.: «Hoc ipso quod primum, est perfectissimum in producendo, fontalissimum in emanando et fecundissimum in pullulando [...]: hinc est quod primum principium, quia primum, est aeternum cum plenitutidine omnium nobilium conditionum; quare in ipso, in quantum primum, status est rerum et comprehensionum». 
deve essere in grado di compierlo ${ }^{27}$. Partendo dall'aspetto dinamico del processo trinitario, Bonaventura risolve le Persone nelle relazioni che istituiscono tra loro, mettendo in luce come determinante l'azione generativa. Il primato del Padre è tale non solo rispetto alle altre due Persone ma in rapporto a ogni essere concepibile, essendo egli l'origine assoluto in ogni ordine di pensiero. Ribadendo tale primità, propria del Padre, Bonaventura annota che "in Lui vi è la pienezza originale (fontalis plenitudo) da cui scaturisce la produzione di tutte le persone. Per cui, dato che il non esser fatto (innascibilitas) include questa proprietà, si dice che tale proprietà si identifica con la nozione stessa di padre" ${ }^{28}$. Alludendo all'origine del creato Bonaventura sottolinea che questo, se è opera della Trinità, è anzitutto opera del Padre ${ }^{29}$. "Poiché il Padre produce il Figlio e per mezzo del Figlio e con il Figlio produce lo Spirito Santo, perciò Dio Padre per mezzo del Figlio con lo Spirito Santo è Principio di tutto il creato. Infatti, se non li producesse dall'eternità, non potrebbe, mediante loro, produrre nel tempo. A motivo quindi di questa produzione, rettamente si dice che nella Trinità c'è la fonte della vita" ${ }^{30}$. Accennando alla sacralità della vita e alla nobiltà degli esseri creati, Bonaventura precisa che il Padre "nel generare suo Figlio ha anche disposto eternamente ciò che avrebbe prodotto nel tempo, e che poi ha prodotto nell'ora fissata. Perché in questa disposizione eterna, non solo ha determinato che quelle cose sarebbero state prodotte, ma anche quando sarebbero state prodotte" ${ }^{31}$.

\section{B. L'INDOLE OBLATIVA DELla POTENZA GENERATIVA DEL PADRE}

Oltre alla potenza generativa, il Padre ne conferma l'indole espansiva di calore e di luce, riconducibile alla categoria del 'bene'. Alla fonte della Trinità come delle creature la diffusività del bene. Con Riccardo di san Vittore

${ }^{27}$ Summa Theologiae II-II, q. 25, art. 4: “Amor est virtus unitiva; unicuique autem ad seipsum est unitas, quae est potior unione. Unde sicut unitas est principium unionis, ita amor quo quis diligit seipsum, est forma et radix amicitiae, in hoc eim amicitiam habemus ad alios, quod ad eos nos habemus sicut ad nos ipsos; dicitur enim in IX Ethic. quod amicabilia quae sunt ad alterum veniunt ex his quae sunt ad seipsum". Alcuni refusi n. 50: raison, prospective, selon.

${ }^{28}$ Ibid., resp. 4: «Ratio primitatis principaliter residet circa personam Patris, ratione cuius est in ipso fontalis plenitudo ad productionem omnium personarum. Unde et quia innascibilitas hanc proprietatem includit ideo dicitur esse proprietas, qaue est notio patri».

${ }^{29}$ Collationes in Hexaëmeron (= Coll. Hex.) XIII, n. 13: «Pater enim ab aeterno genuit Filum similem sibi et dixit se et similitudinem suam similem sibi et cum hoc totum posse suum; dixit quae posset facere, et maxime quae voluit facere, et omnia in eo expressit, scilicet in Filio suo seu in isto medio tanquam in sua arte».

${ }^{30}$ Ibid., resp. 7.

${ }^{31}$ II Sent., d. 13, dub. 3. 
Bonaventura nota che là dove c'è il sommo bene c'è bontà perfetta e cioè comunicazione totale. La natura divina è pienamente feconda nella persona del Padre e ciò in linea con la sua proprietà personale. Il nome che conviene al Padre è rivelato dal Figlio ed è quello di Buono: "Dionigi seguendo Cristo, ci dice che Bonum è il primo nome di Dio" 32 . L'essere trinitario e temporale è da ricondurre al bene, nel senso che l'essere è voluto e dunque è più che essere, nel senso che porta in sé l'onda effusiva propria di ciò che per natura si diffonde e si dona. Rientra nella logica dell'oblazione ciò che viene sviluppato in riferimento alla creazione, in quanto esprime la generosità assoluta dell'amore divino che ha la fonte originaria nel Padre e la sua massima manifestazione trinitaria nello Spirito. Quale dunque l'indole dell'essere e del pensare di Dio? L'indole è oblativa. A sostegno due ragioni. La prima richiama l'esistenza della terza persona della Trinità - lo Spirito Santo - essenzialmente dono e donazione, come congiunzione del Padre e del Figlio di cui è il frutto. Bonaventura ama sottolineare che lo Spirito non è il frutto dell'amore del Padre e dell'amore del Figlio, come per lo più si ritiene, lasciandosi prendere sotterraneamente dalla logica in qualche modo egoistica. Lo Spirito è la risultante dell'amore del Padre non per sé e di sé ma per il Figlio, e dell'amore del Figlio non di sé e per sé, ma per il Padre, e dunque essenzialmente dono e donazione ${ }^{33}$. Se Dio è essenzialmente dono mutuo e dunque oblazione di sé all'altro, il creato, che ha voluto, va pensato come "dono" e la sua volontà come essenzialmente "oblativa". La seconda ragione è da trarre dalla constatazione che Dio è sommamente perfetto e dunque non pressato ad agire da finalità estrinseche. Al Padre, dunque, la potenza creatrice, animata dalla logica oblativa. Ed è secondo tale appropriazione che professiamo la fede, secondo il dettato della liturgia, "in Dio, Padre onnipotente, creatore del cielo e della terra". Il mondo, dunque, rinvia a colui che l'ha creato con un atto perfettamente libero e gratuito.

Ebbene, il parricidio che l'età moderna mette in atto consiste nella contestazione di questa impostazione del problema sia di Dio che delle creature, tutta ispirata alla volontà liberale del Padre, rispetto a cui risulta del tutto impotente e fatua la ragione e il suo potere. Il mistero trinitario

${ }^{32}$ Itin. V, n. 2: «Dionysius sequitur Christum dicit quodbonum est primum nomen Dei». In I Sent. (d. 22, a. u., q. 3, resp.) aveva precisato che «Dionysius videtur velle quod illud nomen 'bonum' solum sit proprium et principale».

${ }^{33}$ I Sent., d. 13, a. u., q. 1. Nel Breviloquium, pars I, cap. IV, n. 9: «Per hunc modum, cum proprium sit Spiritus Sancti esse donum, esse nexum seu caritatem amborum, esse etiam Spiritum sanctum; donum nominat ipsum ut datum voluntarium; caritas sive nexus, ut datum voluntarium et praecipuum $[\ldots] »$. 
come l'atto creativo sono da angolazioni diverse senza ragione, nel senso che trascendono la logica della ragione, incapace di sopportare il peso della suprema perfezione del circolo trinitario e della radicale gratuità del mondo. L'età moderna non contesta frontalmente tale linea perché autoritaria e monodimensionale. L'età moderna cambia registro, apre un altro percorso, e cioè far valere anzitutto i diritti della ragione. Non si tratta della contestazione della 'dominica potestas ${ }^{34}$ percepita come mortificante. Certo la politica ecclesiale è senz'altro di carattere dominatorio e possessivo - non senza un sottofondo polemico la Scuola francescana rivendica come tratto specifico del messaggio cristiano la libertà creativa di segno oblativo, ben consapevole del carattere sovrastrutturale di carattere chiaramente politico un certo stile ecclesiale. E tuttavia, l'età moderna non è questa politica che contesta, quando l'impianto valoriale e dunque quella dimensione oblativa e gratuita, voce nascosta di tutto ciò che è. L'età moderna apre un'altra epoca.

\section{INDOLE EPIFANICA DELLA TEOLOGIA FRANCESCANA}

E' significativo che alla fonte dello scenario teologico il francescano ponga la libertà assoluta di Dio. In fondo la "Scriptura sive theologia" si risolve nel come Dio si manifesta nel tempo, sua culla. Bonaventura non si chiede prevalentemente "quis" o "quid", ma soprattutto "quomodo" il Dio trino si offre 35 . "Descrivere Dio che appare trinitariamente al mondo [...] tale la doppia originalità, teologica e fenomenologica, dell'impostazione bonaventuriana" 36 , preoccupata di fare apparire lui stesso come la cosa stessa, cioè come Padre, Figlio e Spirito Santo. Certo l'apparire trinitario divino non si dà immediatamente, ma si consegna attraverso una molteplicità di segni, che non è agevole intendere. E' la polisemia scritturistica, fonte di una bibliografia inesauribile, attenta a tematizzarne la dimensione cosmica, non estranea alle espressioni religiose di popolazioni con altre culture e altra storia. Il richiamo è a quella ricchezza metaforica che qualifica le pagine bibliche, o anche a quei modi di manifestarsi di Dio, di cui lo sguardo di Francesco era colmo ${ }^{37}$. Elemento speculativo ed elemento simbolico intrecciati,

\footnotetext{
${ }^{34}$ Per bene intendere la portata della nomenclatura - paterfamilias, patria potestas, dominica potestas - cf. CANTARELla, Come uccidere il padre, 20-22.

${ }^{35}$ Armaury Begasse DE DHAEM, "Scriptura sive theologia o come Dio viene all'uomo. Il proprium di San Bonaventura secondo É. Gilson ed E. Falque", in Trilogia bonaventuriana, cur. Carmelo Pandolfi e Rafael Pascual (Roma: IF Press, 2020), 680.

${ }^{36}$ Ibid., 681.

${ }^{37}$ Icel., cap. 29, n. 82: «Enimvero cum a quodam fratre quidam die fuisset interrogatus, ad quid etiam paganorum scripta et ubi non erat nomen Domini sic studiose colligeret, respondit dicens:
} 
memori che "il Verbo incarnato ci ha insegnato la scienza della verità secondo il triplice modo della teologia - simbolica, propria e mistica - affinché con la simbolica usiamo rettamente delle cose sensibili, con la propria usiamo rettamente delle cose intelligibili, con la mistica siamo rapiti agli eccessi sovrarazionali" ${ }^{\prime 3}$. La mediazione del teologo è preziosa, purché fedele alla descrizione del dato biblico ${ }^{39}$ e rispettoso di Dio soggetto, mai oggetto ${ }^{40}$, nel senso che è lui che parla e opera ${ }^{41}$. E' il Dio di Abramo, il Dio di Isacco, il Dio di Giacobbe, non il Dio dei filosofi, mortificato nella rete della nostra logica. L'anima volontaristica della proposta scotista e occamiana trova in questo contesto lo spazio più conveniente, e cioè è Dio che si autorivela come vuole, non come noi desideriamo, ben oltre la barriera della ragione umana. Da qui la preparazione alla lettura del testo, e dunque il senso della nostra dipendenza: attendere che Dio parli o faccia o appaia. Ciò che ha fatto e detto poteva non dirlo, non farlo. Nulla è deducibile da qualcos'altro. All'origine la libertà, attivata dalla gratuità. Il francescano è il povero che attende, ascolta, medita e ringrazia. La sua "speculatio" è quella "del povero nel deserto", dove Dio si esprime imprimendo il suo sigillo "tam mente quam carne" ${ }^{\prime 2}$. L'esistenza si ritrova illuminata in tutte le sue possibili espressioni, ben oltre la trama razionale e lo spazio empirico. La teologia è un percorso, un cammino, un 'itinerarium', lungo il quale ammirare le meraviglie del signore - mirabilia Domini - guidati dalla teologia affettiva che "silentium omnibus aliis potentiis imponit" messo in luce dalle proposte filosofiche che condividono l'atto creativo divino. Nell'ottica francescana si impone con prepotenza il problema del senso, grazie al presupposto che protagonista della scena è Dio che ha scelto e realizzato ciò che poteva non scegliere e non realizzare, per cui diventa

\footnotetext{
"Filii, quia ibi litterae sunt, ex quibus componitur gloriosissimum Domini Dei nomen. Bonum quoque quod ibi est non pertinet ad paganos, neque ad aliquos homines, sed ad solum Deum, cuius est omne bonum"».

${ }^{38}$ Itin. I, n. 7.

${ }^{39}$ DE DAHEM “Scriptura sive theologia”, p. 672, dove riporta l'annotazione di E. Falque (Saint Bonaventure et l'entrée de Dieu en théologie, Paris, Vrin 2000, p.169, nota 3), e cioè che "rispetto a Tommaso che distingue il processo (teologico) dell'analogia e l'uso (unicamente scritturistico) della metafora, Bonaventura [...] si sforza [...] di tradurre secondo la speculazione - come specchio del mondo o speculatio - il movimento stesso della Scrittura [...] la speculazione come modo della sapienza cristiana".

${ }^{40}$ Brev., Prologus, 2, 2.

${ }^{41}$ Brev., Prologus, 4, 5.

${ }^{42}$ Lignum vitae, Prologus, n. 1.

${ }^{43}$ Coll. Hex. II, n. 30.
} 
conseguente la domanda: perché ha fatto ciò che poteva non fare? quale il compito che ci assegna? Non è arduo cogliere quella vena di carattere dialogale che esige ascolto e servizio, per liberare il terreno da quelle scorie oppressive che si sono accumulate nel tempo e ritrovare genuine e prepotenti le istanze che ne denunciano il carattere illusorio. Viene in discussione la filosofia sia moderna che contemporanea che affronta la prospettiva con strumenti del tutto estranei alla logica dell'atto creativo di segno oblativo. E' la filosofia del diritto-a-essere. La proposta francescana vuole che il problema del senso dell'esistenza vada affrontato con gli stessi strumenti con i quali è emerso, e cioè alla luce della volontà oblativa del padre, perché rinvia a una prospettiva ben altra rispetto a quella vincente di carattere conflittuale, ispirata invece al primato del potere, propria dell'età moderna. Alla luce del primato del servizio le norme interumane relative all'organizzazione effettiva della convivenza non sono caricate del peso dell'Assoluto, ma lasciate alla sensibilità e cura degli uomini nella direzione dell'affratellamento. "L'Assoluto si applica solo all'esigenza morale, che dovrà guidare tutte le regole giuridiche" 44 , identificabili con la disponibilità ad ascoltare e a sostenere, nel quadro di una relazione dialogale. E' un atteggiamento di principio cui il cristianesimo non ha mai smesso di guardare, custodendone la trascendenza sulle forme specifiche di convivenza.

\section{IL PADRE E LA PLURALITÀ DELLE CULTURE}

La figura del padre non è ingombrante né impositiva, non perché non abbia tratti specifici, ma perché persegue l'obiettivo primario, che è quello di rianimare tutte le culture nel nome dell'affratellamento universale ${ }^{45}$. La sua proposta prende il volto di tutte le culture, impegnata a contrastarne la piega dominatoria, non ad alterarne il volto specifico.

\footnotetext{
${ }^{44}$ Rémi Brague, Il futuro dell'Occidente, trad. Adriana Soldati (Milano: Rusconi, 1998), 162.

${ }^{45}$ FrUGONI, Un presepio con molte sorprese, 32: «Francesco oppose un silenzioso e deciso rifiuto alla violenza in nome di Dio, senza attaccare la Chiesa ma, come era suo costume, offrendo come esempio il suo dissonante comportamento, cioè una letterale adesione al comando di Cristo di amore e di pace. E' stato notato come negli scritti di Francesco manchino del tutto i termini miles, militia, militare e perfino quei termini che potrebbero essere collegati ad una metaforica lotta contro il maligno: per Francesco conta non combattere, ma servire Deo». Cf. Robert PACIOCCO, "Sub iugo servitutis'. Francesco, i Francescani e la 'militia Christi", in "Militia Christi" e Crociata nei secoli XI-XIII. Atti della undecima Settimana internazionale di studio, Mendola 28 agosto - 1 settembre 1989 (Milano: VeP, 1992), 699-715.
} 


\section{A. LA LIBERTÀ DEL PADRE E LA PLURALITÀ DELLE CULTURE}

Traduzione dello spirito evangelico, la figura del padre si incarna nelle culture, preservandole e insieme rianimandole. In quest'ottica si illumina la portata del conflitto papa-imperatore, e cioè in merito a quella singolarità che vede da una parte il tratto religioso e dall'altro la libertà civile, non subordinati l'uno all'altra ${ }^{46}$. Lord Acton scrive: "A quel conflitto durato 400 anni dobbiamo la nascita della libertà civile, in quanto il papa ha impedito all'imperatore di mettere le mani sul religioso e l'imperatore ha impedito al papa di metter le mani sul politico" ${ }^{47}$. Senza il vincolo politico la proposta religiosa si è potuto trasmettere ad altri ambiti culturali, consentendo all'impianto politico e civile di svilupparsi cercando al proprio interno le leggi più consone, alla luce della logica oblativa della paternità francescana. E' qui la potenza dell'essere-dono, voce che chiama a dar corpo alla propria creatività per smuovere le acque, facendo fronte alla situazione problematica del momento. La dimensione trascendente, che ci interpella e a cui occorre rispondere, riguarda la nostra creatività contro la piega al compromesso e al disimpegno, mettendo in atto la propria forza esplorativa, relativa alla situazione in cui si vive, con una risposta che confermi l'originario atteggiamento creativo. Nel contesto della paternità francescana di assoluto si dà solo la volontà di libertà di segno oblativo contro l'indifferenza e l'egoismo, non questa o quella cultura, questo o quello stile, che invece cambiano nel tempo. E' l'imperativo categorico francescano, articolabile secondo le infinite modalità della vita.

\section{B. AGLI INIZI DELLA STORIA DELL’UOMO}

L'oscuramento di questo scenario con il passaggio dell'essere come dono all'essere come diritto rimanda agli inizi della nostra storia, quando il cammino ha assunto una tonalità conflittuale e dominatoria. Qualunque sia l'indole di questo cambio qualitativo, ciò che occorre ammettere è che gli è connaturato il senso del divino - è diffusa l'idea dell'innatismo delle idee divine o dell'illuminazione divina, grazie a cui l'umanità non si è lasciata assorbire entro il circuito del divenire, facendo fronte alla forza sovrastante della natura con i suoi sconvolgimenti. Il contenuto del dialogo del serpente con Eva nell'Eden conferma questa lettura. La consumazione della disobbedienza, intesa come tentativo di impadronirsi del divino, rappresenta quel momento

\footnotetext{
${ }^{46}$ E' in quest'ottica che va rivalutata la personalità di Frate Elia, amico di Federico II, a sua volta fedele alla Chiesa, ma non suo suddito.

${ }^{47}$ BRAGUe, Il futuro dell'Occidente, 165.
} 
della storia dell'uomo che oscilla tra fedeltà e rifiuto della logica originaria. Il cammino effettivo della preistoria come poi della storia è segnato da infinite traversie provocate dal passaggio dalla donazione all'autoaffermazione, dall'atteggiamento di gratitudine all'atteggiamento conflittuale. E' il prender forma della cultura difensiva e offensiva di segno immanentistico ai fini della sopravvivenza, durante la quale affiorano tracce di quel disegno - il richiamo è alla plurimillenaria avventura, mitica e selvaggia, umana e divina, che abbiamo alle spalle. Decisivo il passaggio dal mito al logos, con cui inizia l'epoca dell'affermazione della ragione, titolare di un sapere "epistemico", veritativo. Il suo tratto tipico sarà costituito dal carattere potestativo - sapere è potere. E' ovvio che l'interrogativo che si impone non riguarda il potere del sapere, ma l'uso, la sua messa in atto, la direzione lungo la quale si è venuta configurando l'umana convivenza. L'interrogativo è: quale modello di uomo viene emergendo? E qui il dialogo tra la visione tendenzialmente razionalistica del mondo e la prospettiva biblico-cristiana si fa serrato, con lo smarrimento dell'indole gratuita del mondo e con la riduzione dello spazio effettivo di carattere oblativo del nostro potere, a causa del predominio dell'autonomia della ragione, presto intesa come autosufficienza. Si intravede sullo sfondo il padre-padrone, colui che si serve del sapere ereditato come dello strumento più raffinato e insieme più efficace a favore dei suoi progetti di segno totalitario.

\section{RETTIFICA DEL RUOLO DELL'UOMO}

Ebbene, il contributo della scuola francescana è costituito dalla denuncia di questo oblio e dalla rettifica del nostro ruolo, attraverso il recupero del carattere oblativo dell'essere. Tale contributo non verrebbe adeguatamente inteso se non mettessimo al primo posto la dimensione gratuita del disegno divino e non puntualizzassimo che la presa di coscienza di tanta grandezza, garantita dalla redenzione cristiana, comporta l'affermazione di uno stile ispirato alla radicale gratuità, quale interpretazione adeguata del suo contenuto. In breve, l'originalità del contributo francescano sta nel sottolineare sia l'indole del disegno e sia la nobiltà dell'uomo, premesse di un atteggiamento non da esecutore, ma da protagonista. Dall'insieme risulta che il nostro essere è fatto non per prendere ma per dare, non per accumulare ma per distribuire, come conviene al gran signore, munifico e sensibile, cosciente della sua dignità e del suo ruolo. Alla fonte del pensare e dell'operare non il turbamento ma lo stupore, non la concupiscenza ma la gratitudine, nella consapevolezza che iniziamo la nostra esistenza da beneficiari del mondo, in 
totale gratuità. In fedeltà a tale logica, si è invitati a impiantare l'esistenza all'insegna della gratitudine, a interpretare il reale come dono, e ad assumere il ruolo di benefattore della comunità. Il mondo non è neutro, di nessuno e a nessuno affidato. La prospettiva biblico-cristiana pone al centro un protagonista - l'uomo - impegnato a generare gratitudine per ciò che fa e per come fa ciò che fa. Il mondo non è un enigma da sciogliere né una miniera di risorse da sfruttare. E' uno scrigno ricco di segreti da decifrare per accrescere la gioia di essere e condividerla ampliando il raggio dei fruitori. Siamo invitati ad assumere uno sguardo di assoluta benevolenza rispetto al tempo e a ciò che accade, condividendone il peso ${ }^{48}$. La visione filosofico-teologica dell'essere, dono dell'Altissimo, non è senza ripercussioni, dimostrandosi cifra di valori decisivi ai fini della valutazione complessiva del reale.

\section{L'INFINITO AL SERVIZIO DEL FINITO}

L'esaltazione francescana dell'uomo vince l'immaginazione la più ardita. Pensato assieme al Verbo di Dio come primum volitum, l'uomo si impone come la creatura prediletta di Dio, non servo né strumento d'alcuno o d'alcunché.

\section{A. L'ESPLOSIONE DELL'OBLAZIONE}

La teologia dell'incarnazione del Verbo è da intendere come una grande onda di amore riversata nel tempo. In fondo è questa la predestinazione di Cristo e dell'uomo alla gloria, anteriore e indipendente dall'avventura errabonda di Adamo ${ }^{49}$. La relazione tra Dio e l'uomo è in cima a tutta la costruzione. Quale l'obiettivo della passione e morte di Cristo? E' l'elevazione dell'uomo, il ritrovamento della sua dignita ${ }^{50}$. La passione e morte di Cristo costituiscono un supremo e libero atto di amore di Dio per la sua creatura, perché abbia l'energia necessaria per non disattendere il suo disegno di gloria, recuperando la libertà creativa di segno oblativo, nel contesto del bello, del vero, del buono, riscoperti e riproposti pur nell'opacità della storia. La perfezione che meglio esprime il

\footnotetext{
${ }^{48}$ Brev. II, n. 4: «Haec igitur sensibilis corporalium machina est tanquam quaedam domus a summo opifice homini fabricata, donec ad donum veniat non manufactam in caelis».

${ }^{49}$ Scotus, Ord. III, d. 32, q. u., n. 5: «[...] qui enim amat se ordinate, et per consequens non inordinate zelando, vel inviendo isto modo, secundo vult habere alios diligentes: et hoc est velle alios habere amorem suum in se, et est praedestinare eos, si velit eis hoc bonum finaliter».

${ }^{50}$ Scotus, Lectura III, d. 7, q. 1-2 (appendix, XX, 446): «Veruntamen si non fuisset honmo lapsus, non fuisset Christus homo natus ad patiendum et sustinendum passiones et ignominias: haec enim sustitnuit propter reparationem naturae lapsae».
} 
volto di Dio, che ha avviato questa avventura, è la libertà creativa, riflessa in un panorama che Egli stesso ha ammirato - "valde bonum" - e confermato con la proclamazione solenne dall'alto della croce: "tutto è compiuto"! Ecco il nodo dei misteri - l'Infinito che associa a sé 1'uomo, Dio che ama le sue creature al punto da mettersi a loro disposizione. E' l'immagine più alta della paternità francescana, incarnazione della logica della gratuità, da attivare condividendo tale logica oblativa e confermandone la grandezza ${ }^{51}$.

\section{B. L'UOMO COME "PRIMUM VOLITUM"}

Nell'ottica francescana l'uomo è sin da principio unito al Verbo incarnato, che è "summum opus Dei" 52 , summum bonum in entibus ${ }^{53}$, summum bonum gratiae $^{54}$. Si tratta del primato dell'umanarsi del Verbo - la creazione non è anteriore all'Incarnazione, ma successiva. Prima l'Incarnazione - il disegno dell'uomo-Dio - e poi come suo ambiente la creazione. Il pilastro dell'intera costruzione è, infatti, il Verbo «primogenitus omnis creaturae» (Col 1,15) - il Dottor serafico non esita a parlare del creato come del "templum Dei" e ad affermare che "Verbum divinum omnis creatura, quia Deus loquitur", confermando che le creature sono l'alfabeto del discorso di Dio. Duns Scoto, da parte sua, distingue chiaramente l'incarnazione dalla creazione ritenendo questa funzionale all'Incarnazione del Verbo, "non occasionata [da qualcosa di contingente], ma dall'eternità vista immediatamente da Dio come un fine [...]. Dunque questo fu l'ordine nella previsione divina: in primo luogo Dio comprese sé stesso sotto il carattere del sommo bene [...]; in quarto luogo previde che [gli uomini] sarebbero caduti in Adamo; in quinto luogo, preordinò ovvero previde il rimedio, come cioè essi sarebbero stati redenti grazie alla passione del Figlio. In tal modo il Cristo nella carne, come anche tutti gli eletti, era previsto e predestinato alla grazia e alla gloria prima che fosse

\footnotetext{
${ }^{51}$ Claude Tresmontant, La Christologie du B. Jean Duns Scot, l'Immaculée Conception et l'avenir dell'Eglise. Note complementaire à propos du peché originel (Paris: François-Xavier de Guibert, 1996), 46: «La raiuson d'être du Christ est donc beaucoup plus que de réparer la faute originelle, puisque c'est in lui, par lui, avec lui qu'est en realité créé l'Homme veritable que Dieu en visage depuis les origines ou avant les origines de l'univers. Combien nous sommes loin de la perspective augustinienne, reprise et aggravée par sain Anselme de Conterbury, reprise et aggravée encore plus par Martin Luther, Calvin et Jansenius, - prospective selon laquelle la raison d'être du Christ est seleument ou du moins principalement d'obtenir à notre place le pardon de Dieu pour la faute originelle et de réparer les dégâts».

${ }^{52}$ Reportata Parisiensia (=Rep.) III, d. 7, q. 4, n. 4.

${ }_{53}$ Ord. III, d. 7, q. 3, n. 64 .

${ }^{54}$ Ord. III, d. 13, q. 4, n. 54.
} 
prevista la passione di Cristo quale medicina contro la caduta, cosi come un medico vuole la salute dell'uomo prima di prescrivere un medicamento per la cura" 55 . In quest'ottica la creazione è l'ambiente del Verbo che si incarna, o, come si legge nella Lettera agli Ebrei $(3,6)$, è l'abitazione che il Verbo si costruisce e in cui si insedia "tamquam filius in domo sua". Cristo non è il "nuovo" Adamo, ma il "primo" Adamo, è il Figlio dell'Uomo, nel senso che il Verbo ha dall'eterno in sé il volto di ogni uomo. E san Francesco: "Considera, o uomo, in quale sublime condizione ti ha posto il Signore Dio, poiché ti ha creato e formato a immagine del suo Figlio diletto secondo il corpo (ovvero del Verbo fatto uomo $^{56}$ ) e a similitudine di lui secondo lo spirito" 57 . Su tale sfondo cristologico, non ci sorprende che le creature abbiano nostalgia della patria divina e che il loro tempo sia aggrappato all'eterno - l'Incarnazione segna il contatto tra l'eterno e il tempo. $\mathrm{Ne}$ consegue che la fedeltà a tale logica esige che la nostra condotta non sia mossa dall'onda possessiva e dominatoria che ci abita, ma si lasci guidare da quell'onda oblativa che emana dall'Incarnazione del Verbo, riversata su di noi grazie alla sua opera redentiva. Se l'Assoluto è "eterna attività creatrice", la libertà è lo spazio attivo dell'uomo, chiamato ad agire entro l'ambito della creazione, voluta dal Verbo come il suo trono nell'eterno e come la nostra casa nel tempo, da custodire con intelligenza e abbellire con passione. Dunque, la libertà creativa in principio, la cui realizzazione è opera di quell'onda d'amore, sostanza del bene, anima del vero. È il processo creativo dell'amore oblativo di matrice francescana: "Dio è amore non perché ama il Bene [...]; il Bene è 'effetto' dell'Amore, non viceversa. [...] La radice dell'amore è nella soggettività, non nell'oggettività" ${ }^{\prime 8}$ e riguarda la totalità del soggetto ${ }^{59}$. È quanto ribadisce in più circostanze Duns Scoto, per il quale "nullum aliud bonum (praeter seipsum) quia bonum ideo amatum ab illa voluntate, sed e converso" 60 . E' il principio dell'etica secondo cui il volere è attivato dall'amore, costitutivo del bene, sicché ciò che non è voluto non rientra nell'ambito del bene. Siamo fuori dell'ottica del Dio "padrone", cui tutto dovrebbe far ritorno perché di tutto l'artefice. Il disegno originario

\footnotetext{
${ }^{55}$ Ord. III, q. 3, n. 58.

${ }^{56}$ Scotus, Quaestiones Quodlibetales, q. XIX, n. 2: "Verbum factum est, ubi caro pro homine ponitur".

${ }^{57}$ Francesco, Ammonizione V, FF 153.

${ }^{58}$ Santiano CAVAciuti, Libertà e trascendenza (Soveria Mannelli: Rubbettino, 2018), 46.

${ }^{59}$ Bonaventura, I Sent., d. 45, a. 2, q. 1, concl.: «Ratio causandi est bonitas, et in ratione effectivi et in ratrione finis. Nam bonum dicitur diffusivum sui, et bonum est propter quod omnia».

${ }^{60}$ Scotus, Ord. I, d. 41, q. u., n. 54.
} 
di Dio è invece contrassegnato dalla logica oblativa, intesa come perseguimento del bene per il bene. Il volontarismo francescano con le sue molte implicazioni filosofico-teologiche è il compendio del primato della libertà sulla necessità, dell'oblazione sulla rivendicazione, dell'essere voluto sull'essere dovuto. E' lo stile dell'uomo che il francescano vuole che cambi, e cioé da padrone, che domina, a signore, che ammira e serve.

\section{LA DIMENSIONE OBLATIVA ANIMA DELLA LIBERTÀ CREATIVA}

Si comprende in quest'ottica il recupero della libertà creativa, attivata secondo la logica oblativa dell'essere. E' sommamente benefica l'onda causale del padre, autentica non quando è priva di ragioni, ma quando è rivelativa di tutto se stesso che dona donandosi. Per questo, nonostante che abbia intrinseca al proprio essere la vocazione alla realizzazione, la sua potenza causale, essendo una sorta di causalità "potenziale", in analogia a ogni causalità potenziale come, ad es., il camminare o il sentire che hanno senso in quanto si cammina o si sente, si ritrova irretita nelle maglie della concupiscenza, da cui, anche se a fatica, va liberata ${ }^{61}$, perché l'amore creativo di segno oblativo prevalga oltre l'ambito specifico di un particolare dono, attraverso cui pur si concretizza. E' il nucleo qualificante dell'itinerario da intraprendere, mettendo in atto la libertà dell'intelligenza, segnata dalla dimensione verticale, che accompagna e sostiene il passaggio dalla ragione calcolante all'intelletto meditante. E' la libertà dell'intelligenza, che consente al padre di accogliere l'evento nel quale si annuncia il non-conosciuto. Senza libertà si rimarrebbe prigionieri del proprio mondo, ricco o povero che sia. "C'è povertà di libertà, prima ancora che pochezza intellettuale - scrive il bonaventuriano Malaguti - in chi non sa levarsi al di sopra delle acquisizioni sociali; in chi non prova entusiasmo per la grandezza di una scoperta se essa mette in crisi le presunzioni scientifiche comunemente accettate; in chi rifiuta lo splendore di un messaggio perché lo vede irriducibile alle misure dell'esperienza abituale" ${ }^{\prime 2}$. E' la disponibilità al nuovo la fonte dell'atteggiamento dialogale padre-figlio, da intendere come fiducia nell'orizzonte dell'altro e insieme come ricerca di adeguati criteri di intelligenza ai fini della sua corretta valutazione. Solo chi è libero può uscire dalla caverna dell'io, mettersi in ascolto dell'inaudito e lasciarsi inebriare dai raggi di una luce imprevista

\footnotetext{
${ }^{61}$ Bonaventura, Brev. II, n. 9: «Cum de libero arbitrio loquimur, non de parte animae loquimur, sed certe de tota».

${ }^{62}$ Maurizio Malaguti, La metafisica del volto. Una lettura di Dante (Milano: Edizioni Biblioteca Francescana, 2020), 4.
} 
e calda. Inoltre, la libertà trova respiro nel bene e nel bello, non intesi in senso utilitaristico - il bene che corrisponde ai miei bisogni o il bello che soddisfa il mio gusto - ma come qualità fruibile, grazie a cui cambia lo sguardo e l'occhio diventa penetrante. Così, ad es., "l'intuizione che dà origine a un poema nasce nella libertà della mente che elegge lo splendore dell'eroismo, o l'amabile chiarezza della fedeltà o la ricchezza segreta della saggezza quali principi che rendono desiderabile l'esistenza. Non si può parlare dei principi qualificanti se non a partire dal significato del dono grande e terribile della libertà" ${ }^{63}$. La libertà va collocata nel cuore dell'essere, all'interno del quale costituisce una sorta di spartiacque. Tutto ciò che è senza libertà può essere spiegato nei termini della pura funzionalità, dei giochi di forza, che danno origine al vasto comporsi di realtà semplici o complesse. Mentre tutto ciò che è frutto della libertà si spiega facendo ricorso alla logica della qualità, passione e luce dell'agente, espresse nella trasfigurazione oppure nella deturpazione del volto, quale traccia indelebile della nostra figliolanza di Dio. E' la libertà la premessa insostituibile dell'amore, come dell'odio. "Nessuno potrebbe amare senza libertà. Inteso come libero protendersi dell'esistenza verso la sorgente di ogni bene, l'amore svela al suo interno una sorta di filigrana luminosa attraverso la quale l'intelligenza si innalza verso la trascendenza" ${ }^{94}$. Il fascino sensuale o la vanità del prestigio cedono sotto il potere dell'amore che rende possibile al padre il rapporto con ciò che nel figlio è ancora inattuale, che però intravede e che aiuta a far nascere. L'amore della verità implica il rallegrarsi del fatto che questa si dia, e che possa nascere in forme nuove. In breve, la libertà sia come disponibilità al nuovo, sia come espansione nel territorio del bene e del bello, e sia infine come premessa dell'amore, è possibile intenderla come il tesoro nascosto del padre, dalle mille espressioni, a conferma che egli vive immerso nel mistero della paternità divina. "Le nostre acque - ricorda Bonaventura - non debbono discendere verso il mare morto, ma ascendere verso la loro prima origine" ${ }^{\prime 5}$. E' il percorso che egli segue, non abbandonando il suo soggiorno, ma aprendolo alla luce del sole, perché sia ospitale.

\footnotetext{
${ }^{63}$ Ibid., 69.

${ }^{64}$ Ibid., 90.

${ }^{65}$ Coll. Hex. XIX, n. 15.
} 


\section{LE CRITICHE DEI FIGLI POTENZIAMENTO DELLA PATERNITÀ}

Ai fini dell'interpretazione in chiave elevante e creativa delle molte critiche dei figli nei riguardi del padre - l'intera storia francescana è contrassegnata da contrasti di diversa portata, la cui positività è confermata dalla moltiplicazione dei suoi rami, rigogliosi e innovativi - è necessario assumere come sfondo la dimensione oblativa della paternità. Si tratta in breve di leggere tale storia non alla luce dell'essere come diritto in un contesto rivendicativo, bensì alla luce dell'essere come dono e dunque in chiave creativa, rispetto a cui valutare critiche e dissensi, vissuti anche con asprezza, ma all'interno dell'identica scala valoriale.

\section{A. IL CONTESTO DELLE CRITICHE DEI FIGLI AL PADRE}

E' utile richiamare le coordinate dei due modelli - quello oblativo e quello concupiscenziale, cui si è fatto riferimento fin qui - per meglio sottolineare l'indole della paternità francescana e la sua capacità di assorbire l'onda critica che in certe fasi storiche si è alzata prepotente. Si sa che entrambi i modelli sono costituiti dalla compresenza di volontà e ragione, di libertà e necessità, di singolarità e universalità, di bontà e verità, di autonomia e dipendenza, e che la loro distinzione è da ricondurre alla disposizione di queste componenti, dal momento che la prospettiva biblico-cristiana della paternità nella versione francescana è qualificata dalla libertà creativa di segno oblativo, mentre quella greco-romana dalla ragione potestativa; l'una fa spazio alla creatività come fucina del nuovo per esaltarlo, l'altra fa spazio alla ragione come fucina del disponibile per dominarlo. Sono due prospettive, due logiche, due ontologie. Ebbene, alla luce di tale distanza qualitativa occorre dire che la paternità nella versione francescana prevede al primo posto la volontà, la libertà, la singolarità, la bontà, l'autonomia, e dunque la visione del reale come dono, frutto di una volontà creativa di segno oblativo. La ragione non è assente ma funzionale alla volontà oblativa ${ }^{66}$, come la verità rispetto alla bontà, la necessità rispetto alla libertà, l'universalità rispetto alla singolarità, la dipendenza rispetto all'autonomia. E' questo il contesto da tener presente, alla cui luce la paternità nella versione grecoromana, affermatasi nell'Occidente, risulta fondata sulla ragione - la verità, la necessità, l'universalità, l'ordine, la dipendenza. Sono due prospettive

${ }^{66}$ SCOTUS, Rep. IV, d. 49, q. 2, n. 16: «maioris capacitatis est voluntas quam intellectus, hic et in patria». 
l'una alternativa all'altra, che solo in via eccezionale coesistono, nel senso che solo in determinate circostanze quella francescana prevale ed è ammirata come una luminosa eccezione, mentre quella greco-romana funge per lo più da paradigma della vita quotidiana. Da qui l'audacia del cambio culturale e cioè un modello di paternità di matrice francescana, che reinterpreta la realtà nel suo insieme o almeno ridimensiona la scala dei valori tuttora in auge. La tesi che, sia pure a fatica, emerge in controluce, è che la filosofia occidentale è caratterizzata dal diritto-a-essere, di cui la volontà di potenza di Nietzsche è l'espressione più alta - da qui l'imparentamento tra modernità e post-modernità - e che la contemporanea filosofia del pensiero debole ne è la critica più efficace, restando però all'interno della stessa logica o meglio, condividendo lo stesso punto di partenza, e cioè il diritto-a-essere, accentuato, non attenuato, sorgente sotterranea di tutto il discorso. La proposta francescana suggerisce di modificare tale punto di partenza - non più il diritto-a-essere, ma il dono-di-essere, non il padre che rivendica, ma il padre che accoglie e sostiene. E' all'interno di questa prospettiva che va posta e valutata la storia intricata dei contrasti, anche aspri, tra Francesco e i suoi figli.

\section{B. L'INDOLE DEI MOLTI DISSAPORI}

\section{ALL'INTERNO DELLA FAMIGLIA FRANCESCANA}

Le pagine piuttosto dure, costellate di critiche, che accompagnano la vita di Francesco, sono riconducibili per lo più a una risposta inattesa ed entusiasta alla sua proposta di seguire sine glossa il santo Vangelo di Gesù Cristo. La semplicità e la profondità del progetto emersero presto dando frutti abbondanti, con problemi, più o meno rilevanti, dovuti all'affermarsi di una famiglia poliedrica e numerosa, ben oltre la previsione del suo fondatore $^{67}$. Lo scontro era inevitabile tra quanti sostenevano fedelmente il suo orientamento, pensato su misura di pochi compagni, animati dallo stesso entusiasmo, e quanti invece, alla luce dell'ampliamento della famiglia, proponevano di aprire il quadro programmatico, pur nella persistente fedeltà al Vangelo. Si pensi al capitolo delle stuoie del 1221, per Tommaso da Eccleston con 5.000 frati, per Giordano da Giano con 3.000, in ogni caso con un numero straordinario di partecipanti, ricordato per i contrasti sulla regola

${ }^{67}$ Le GofF, San Francesco d'Assisi, 73: «Francesco non intendeva affatto trasformare i suoi seguaci in un 'ordine', egli non desiderava che riunire un piccolo gruppo, una élite che facesse da contrappeso, mantenesse desta un'inquietudine, un fermento di fronte all'ascesa del benessere. Questo contrappunto francescano è restato un bisogno del mondo moderno, per i credenti come per i miscredenti». 
tra Francesco e i frati più influenti. Anche se "è più probabilmente una fusione di tutti i capitoli di questo periodo, abbellita da questioni sulla povertà degli anni Quaranta e oltre del Duecento"68, questo capitolo non è impossibile pensarlo come la formalizzazione di quello scontro che già da tempo serpeggiava e che andrà ampliandosi sempre più tra la visione iniziale di Francesco e quella che veniva suggerita dai frati consapevoli delle molte istanze della società e dalla variegata composizione della famiglia, in rapida espansione. Si sa che nel 1219 egli lascia la Palestina e torna in Italia per le molte voci di dissenso, concretizzate in proposte, talune stravaganti, da parte di quanti volevano abbandonare il rigorismo iniziale e fare spazio ad altre forme di vita, ritenute più efficaci. Francesco si rende conto di non riuscire a riprendere in mano la situazione "senza l'appoggio della curia pontificia e senza farle di conseguenza delle concessioni. Nelle decisioni prese dal 1221 al 1223 in merito alla riorganizzazione del suo movimento è spesso difficile distinguere le intenzioni del santo da ciò che gli viene imposto" ${ }^{69}$. Egli chiede e ottiene un rappresentante della Santa Sede, il card. Ugolino, "protettore, governatore e correttore della fraternità". Consapevole dell'ampiezza del contrasto e del vento maggioritario nella direzione di atteggiamenti più compromissori rispetto al rigore iniziale, egli decide di dare le dimissioni da guida giuridica della famiglia, cedendo la direzione amministrativa a Pietro Cattani, alla cui morte il 10 marzo del 1221 succederà frate Elia. Con il grave gesto delle dimissioni Francesco si dimostra consapevole che il carisma, di cui Dio lo ha dotato, può fungere da sostegno, non però da fondamento, dell'istituzione. E' il punto d'approdo delle vicende della sua Regola, approvata il 29 novembre del 1223 da Onorio III con molti ritocchi da parte dei frati oltre che dal card. Ugolino - molte citazioni evangeliche sono sostituite da formule giuridiche; soppresso l'articolo che autorizzava i frati a disubbidire ai superiori indegni, come anche le prescrizioni relative alla cura per i lebbrosi; risulta attenuato l'accento sulla rigorosa povertà, come sulla necessità del lavoro manuale o sul divieto di tenere presso di sé i libri. "Con la morte nell'animo, Francesco accettò la regola così deformata. I biografi hanno definito questo periodo della sua vita, verso la fine del 1223 , l'epoca della "grande tentazione". Tentazione cioè di abbandonare completamente il nuovo ordine, se non l'ortodossia"70.

${ }^{68}$ Augustine Thompson, Francesco d'Assisi una nuova biografia (Bari: Edizioni di Pagina, 2016), 280.

${ }^{69}$ Le Goff, San Francesco d'Assisi, 49.

${ }^{70}$ Ibid., 52. 
A conferma della distanza che i figli avevano preso nei riguardi del suo progetto si richiama per lo più il Fioretto VIII intitolato "Come andando per cammino santo Francesco e frate Leone, gli espose quelle cose che sono perfetta letizia" 71 . L'argomento è il netto rifiuto da parte dei frati della sua presenza e delle sue indicazioni di vita. Egli non è riconosciuto come padre. Egli stesso immagina che a notte fonda, nel cuore dell'inverno, bussi alla porta di un convento dei suoi frati, e alle sue insistite richieste di accoglimento il frate risponda: "Vai! Tu sei un semplice e un idiota! Ormai non verrai più da noi! Noi siamo tanti e tali che non abbiamo più bisogno di te!". A buon diritto è stato annotato che si tratta di "una narrazione apparentemente lineare e, alla fine, pacificata, che mostra in realtà divergenze, profondi dissidi e addirittura rifiuti che contrapposero frate Francesco al suo Ordine nel delicatissimo passaggio dall' 'intuizione' di una proposta cristiana alla sua 'istituzionalizzazione" "72. L'altro richiamo riguarda il suo intervento al capitolo delle stuoie, che lo Speculum ${ }^{73}$, servendosi della Compilatio Assisiensis, riporta. Oltre alla fedeltà al Vangelo, egli ribadisce la necessità di condividere la sua "forma vitae" e di non apportare alcuna modifica sia pure tratta da regole della tradizione monastica. E' facile cogliere la distanza tra la linea che i frati intendevano proporre, alla luce anche di altre esperienze religiose, e il richiamo di Francesco alla perfetta osservanza del Vangelo e della povertà - "sine glossa" - lasciando, cioè, fuori qualsiasi altra linea, sia pure più coerente e feconda ${ }^{74}$.

\section{L'AMPLIAMENTO DELL'ORIZZONTE DEL PADRE}

Lo scontro è frontale. Ma non è affatto un parricidio, dal momento che non si tratta di sconfiggere la "patria potestas", quasi che il padre si identifichi con l'autorità nel cui nome imponga la sua volontà. A Francesco turbato per la piega che l'Ordine sta prendendo Chiara ricorda che la famiglia, che ha

${ }^{71}$ De vera et perfecta laetitia, da un manoscritto dell'inizio del XIV secolo, in $F F$ 278: Della vera e perfetta letizia.

${ }^{72}$ Marina Benedetti, “Ma qual è la vera letizia'. Realtà e metamorfosi di Francesco”, in Francesco da Assisi. Storia, arte, mito, cur. Marina Benedetti e Tomaso Subini (Roma: Carocci, 2019), 34.

${ }^{73}$ ANONImo Della Porziuncola, Speculum perfectionis status fratris Minoris (Firenze: Edizioni del Galluzzo, 2006).

${ }^{74} F F$., n. 2011: «Il Signore mi ha detto che io dovevo essere come un novello pazzo in questo mondo, e non ci ha voluto condurre per altra via che quella di questa scienza. Dio vi confonderà proprio per mezzo della vostra scienza e sapienza. Io confido nei castaldi del Signore: per mezzo loro Dio vi punirà. E allora tornerete al vostro stato, lo vogliate o no, con vostra vergogna». 
voluto, non è sua. La paternità è di Dio. O anche, come il Signore sembra che gli abbia suggerito: "Povero piccolo uomo perché ti rattristi? Il tuo ordine non è il mio ordine? Non sono io a esserne il supremo pastore?" E' la dimensione oblativa al centro, da ampliare, intensificare, sostenere. Si tratta di condividere nel profondo l'epilogo pacificante, cui era pervenuto nel colloquio con frate Leone in merito alla perfetta letizia, e cioè, se nonostante il rifiuto e il misconoscimento più rigido da parte dei suoi frati, "avrò avuto pazienza - aveva concluso Francesco - e non mi sarò turbato, in ciò è vera letizia e vera virtù e salvezza dell'anima". E' lo stile dell'oblazione totale, che non può non condividere colui che non vive e opera in nome di Cristo, ma vive e opera "come" Cristo, il quale conferma la sua totale oblazione al popolo ingrato e ribelle. "Vera gioia è quella che assume che il padre sia misconosciuto dai figli e che, di fronte al misconoscimento resiste: una tale persistenza riconcilia veramente il cuore del padre con il cuore dei figli" ${ }^{\prime 7}$. Il contrasto è motivo di dilatazione degli orizzonti, non di rifiuto o di condanna. Francesco si ritira a Greccio dove pregando e meditando trova pace e serenità. Ai primi di giugno del 1224 lascia Greccio per partecipare alla Porziuncola al capitolo generale, durante il quale la distanza rispetto alle origini si approfondisce ulteriormente con i primi passi verso la clericalizzazione ${ }^{76}$. Ed è in questo contesto che va letta la breve lettera che egli indirizza "a frate Antonio, mio vescovo" al quale dice: "Ho piacere che tu insegni la sacra teologia ai frati". Non si sa con quale fatica spirituale abbia scritto "ho il piacere" $"$, dal momento che si tratta di un capitolo che comporta una rilevante ristrutturazione dell'ordine, ben distante dalle forme iniziali di vita, percepita non senza una crescente dilatazione dell'anima, ormai votata alla totale mortificazione dell'io possessivo. Il sigillo divino vien posto nell'eremo della Verna, dove il 14 settembre, durante una visione, si ritrova avvolto dalla luce di un serafino con sei ali e le braccia aperte e i piedi giunti infissi su una croce. Con tale stimmatizzazione Francesco appare "un uomo nuovo

\footnotetext{
${ }^{75}$ Bernard Forthomme, Il canto del corpo ardente. La stimmatizzazione di san Francesco di Assisi (Padova: Messaggero, 2012), 66.

${ }^{76}$ ThOMPSON, Francesco d'Assisi una nuova biografia, 135: «Il 18 marzo 1224, poco più di tre mesi dopo l'approvazione della regola, un gruppo di frati divennero preti, i primi frati a essere ordinati. Il gruppo comprendeva Giordano di Giano, che ci racconta l'evento. Un'altra occasione, che avrebbe facilitato l'ammissione di più candidati con aspirazioni clericali, si verificò nel capitolo di Pentecoste del 1224 giugno, al quale Francesco stesso pare sia stato presente».

${ }^{77}$ Ibid., p. 137: «Ci si potrebbe chiedere se egli fosse consapevole delle implicazioni di questo atto. I frati che vennero dopo videro i risultati di questa decisione, e non tutti ne furono contenti».
} 
di un altro mondo", come dice il Celano ${ }^{78}$. "Età dello Spirito o della libera volontà che approfondisce e supera l'età filiale dell'obbedienza?"79 "Il segno delle stimmate non presuppone la negazione del tempo o della paternità immanente, in vista di una temporalità e di una fedeltà, di una più profonda attestazione dell'esistenza e della fraternità umana, ma anche della fraternità naturale tra gli esseri" ${ }^{\prime}$. L'oblazione di sé è completa, padre della numerosa famiglia dai mille interessi nel nome della libertà dello Spirito, luce del creato. L'esaltazione di Francesco è tale da risultare inimitabile, proiettato in quel futuro intravisto da Gioacchino da Fiore quando la libertà dello Spirito sarà tradotta in effettivo stile di vita dell'intera comunità ecclesiale ${ }^{81}$. E' l'affermazione della dimensione oblativa dell'essere ${ }^{82}$, ben distinta dall'eros che ha segnato di sé la storia, quale guida dell'essere e del pensare occidentale. Il francescano è fermo su questo punto, e cioè la volontà del padre non è riconducibile alla logica potestativa, bensì alla logica oblativa, da intendere come potenza attiva, abitata da quell'onda di vita effusa e moltiplicata. E' in questa logica che matura la libertà francescana della volontà ${ }^{83}$, ben oltre la caverna dell'io, intenta a guardare fuori, oltre e sopra di sé. La creatività le è congeniale, alimentata dall'amore agapico, espressione della libertà in quanto potenza attiva o capacità di fare, ai fini dell'ampliamento dell'orizzonte di vita. E' questo l'obiettivo: realizzare la volontà di potenza non intesa in termini di potere ma di possibilità di fare e di dare, risvegliando energie inattive. Il che comporta un'originale traduzione del nostro essere a immagine di Dio, nel senso che se ne condivide la logica e l'orientamento.

${ }^{78}$ ICel 82, in FF 462; LegM 4, 5, in FF 1072.

${ }^{79}$ FORTHOMME, Il canto del corpo ardente, 91. «La stimmatizzazione non è un semplice avvenimento sociale (feudale), familiare, psicologico o antropocentrico, e neppure cristocentrico, ma è un a vera e propria cifra capace di interpretare, riprodurre e soppesare la potenza della creazione con tutta la sua musicalità: dimensione cosmica in consonanza con la figura isaiana del serafico che vibra e fiammeggia nel tempio, e che è figura dell'universo» (41-42).

${ }^{80}$ Ibid., 90.

${ }^{81}$ E' la prospettiva conciliante di Bonaventura e cioè non siamo ancora all'ordine nuovo degli uomini dell'Ecclesia contemplativa (Coll. Hex. XXII, n. 22).

${ }^{82}$ Quodlibeta XVIII, n. 24 - Scotus, Cuestiones cuodlibetales, ed. Felix Alluntis (Madrid: Biblioteca de Autores Christianos, 1968), 642: «Voluntas autem sola habet indifferentiam ad contradictoria, et talem quod ipsa est sui determinativa ad alterum eorum, ex IX Metaphysicae cap. 4-5, 1048 a 5-15); aliter, simul ageret contraria. Propter hoc igitur quod actus eius sic indifferenter est in eius potestate, licet non nisi supposita intellectione, ideo per se est imputabile agenti».

${ }^{83}$ Ord. III, d. 33, q. u., n. 2: «eius est virtus cuius est per se libere agere». 


\section{LA PATERNITÀ COME AMPLIAMENTO DELLA RESPONSABILITÀ}

La figura del padre, quale pilastro dell'architettura sociale, va ben oltre il breve cono di luce della coscienza. Essa coinvolge tutto l'essere, oltre la ragione in esercizio (cogitatio). E' il padre che si assume la responsabilità di tutto ciò che accade, riconducibile all'immagine del pastore, attento alla sorte dell'intero gregge, impegnato a condividere le disavventure della pecora smarrita e a caricarsela sulle spalle. La responsabilità va oltre la coscienza e dunque oltre la libertà progettuale di segno individuale. E' il modo francescano d'essere padre. La coscienza del proprio io e la connessa libertà creativa rientrano nel circuito della responsabilità, ma senza esaurirla. L'originalità della figura del padre sta nella constatazione che non si dà un'area che gli risulti estranea. Se si parte dal primato della razionalità e si ritiene che le cose ne sono la traduzione, con una propria logica, allora si cerca il titolare, in assenza del quale non c'è che il caso. E' l'ambito delle scienze. Se però si procede dal primato della volontà e si ritiene che le cose sono perché volute - la volontà al primo posto, non la ragione - cambia lo scenario, e cioè l'indole degli eventi e dei fenomeni, e si dilata l'orizzonte, dal momento che gli elementi che compongono l'evento sono assemblati in modo contingente e cioè in modo non deterministico. Il che comporta la necessità di riportare la contingenza dal piano ontologico al piano etico - nel senso che un evento accade, ma poteva non accadere, o accadere diversamente e dunque coinvolge tutti i soggetti che in qualche modo rientrano in quel circuito. Il che presuppone l'interconnessione degli eventi e, data la loro indole contingente, il tentativo di capire come e perché siano accaduti. Il coinvolgimento non riguarda solo gli eventi di cui il padre è attore, dal momento che noi stessi siamo un insieme di elementi non scaturiti dalla nostra coscienza, ma ricevuti e accettati. Infatti, dicendo di sì all'essere e cioè esistendo, noi condividiamo la logica attivata da colui che riteniamo padre di tutto ciò che ci costituisce - ciò che siamo, il nome che portiamo, la professione che ci viene riconosciuta... - ritenendoci responsabili di tutto ciò. Chi di noi non si riconosce in ciò che è, e che tuttavia ha ricevuto? Il padre, che ci ha chiamato all'essere, non ci ha forse immessi nel circuito della vita, sicché tutto ciò che accade rientra nel capitolo della nostra esistenza, coinvolgendola? E' la figura del padre quella che forse più di tutte le altre contribuisce a dare il giusto peso alla dimensione cosmica della responsabilità. Dandoci l'essere, il padre ci dispone a essere corresponsabili di tutte le espressioni della vita. 
E' questo un capitolo qualificante la prospettiva francescana, aperta alle mille voci che si levano dal cuore del creato e s'alzano dall'abisso della storia, che siamo chiamati a raccogliere. Francesco parlava alle creature e considerava fratello e sorelle anche cose inanimate. Non c'è nulla che non abbia un messaggio da trasmettere o una richiesta da avanzare. L'ampliamento dello sguardo e l'accentuazione della dimensione cosmica del nostro essere hanno per obiettivo l'ampliamento dell'arco della nostra responsabilità e quindi della nostra oblazione ${ }^{84}$. Il punto di partenza è la volontà, sia come capacità di iniziare un nuovo capitolo e sia come contributo al miglioramento di ciò che accade. Il che comporta che non c'è nulla che non ci coinvolga, non solo perché abbiamo contribuito, intervenendo o astenendoci, al suo accadimento, ma anche perché ci interpella e ci obbliga a rispondere - quanti eventi, ritenuti casuali, hanno contribuito a modificare la nostra esistenza! Certo, la coscienza vigile al primo posto, non però in quanto prometeica, bensì in quanto attenta a ciò che accade. E' l'antenna, non il tribunale, l'immagine del padre, e cioè la coscienza interessata alla piega che la vita concreta assume riconoscendosi coinvolto e interpellato anche da ciò che sembra estraneo al suo breve circuito.

Da qui la necessità che la coscienza si apra a ciò che accade - ecco la libertà come modo d'essere originario e conferma della dimensione cosmica del suo essere ${ }^{85}$. La responsabilità va ben oltre l'ambito della propria coscienza, nella consapevolezza che ciò che chiamiamo all'essere porta dentro di sé istanze che si impongono anche se non volute. E' l'essere - cioè il fondo abissale della storia - che fa sentire la voce. Si pensi per il mondo classico a Edipo re, responsabile della peste che colpisce Tebe, la sua città, provocata da ciò che egli ignora - l'uccisione del padre Laio e l'unione incestuosa con la madre. Si pensi nell'ambito biblico-cristiano al peccato originale, il cui peso grava su quanti vengono al mondo, ignari di ciò che è accaduto ai primordi della storia. Come è possibile sentirsi colpevole di ciò di cui si è vittima? Quale l'indole dell'orizzonte che si viene definendo?

${ }^{84}$ II Sent., proemium, n. 2: «Fecit igitur Deus hominem rectum, dum ipsum fecit ad se conversum. In conversione enim hominis ad Deum non tantum rectificabatur ad id quod sursum, sed etiam ad id quod deorsum. Homo enim in medio constitutus, dum factus est ad Deum conversus et subiectus, cetera sunt ei subiecta, ita quod Deus omnem veritatem creatam subiecerat eius intellectui ad diiudicandum, omnem bonitatem eius affectui ad utendum, omnem virtutem eius potestati ad gubernandum».

${ }^{85}$ II Sent., d. 14, a. 1, q. 1: «Non habent ipsae creaturae irrationales immediate ad Deum ordinari, sed mediante creatura rationali. Ipsa enim creatura rationalis, quae de se nata est et laudare et nosse et rtes alias in facultatem voluntatis assumere, nata est ordinari in Deum immediate». 
L'uomo è vittima e colpevole a un tempo. Il suo essere è ferito, difettivo, amante di se stesso, e pertanto colpevole, e cioè portatore di una colpa non commessa ma ereditata, quella del ripiegamento di sé su se stesso, di cui il nostro agire è il prolungamento. ${ }^{86}$ Nulla di ciò che accade ci è estraneo. Di tutto dobbiamo portare il peso, come il buon padre rispetto alla famiglia. Ed è appunto in questa direzione l'ammonizione di Francesco a "ricominciar da capo", perché finora "abbiamo fatto poco o nulla". Il francescano è quel padre che si sente responsabile non solo di ciò che ha chiamato all'essere, ma di tutto ciò che accade, perché rientra in quel mondo che è suo dal momento che ad esso ha detto sì, esistendo. Tutto questo "comporta la coscienza che ciascuno di noi è un momento della formidabile e incredibile avventura umana, la quale è un ramo ipersviluppato della formidabile e incredibile avventura della vita, la quale è un ramo ipersviluppato della formidabile incredibile avventura del cosmo" ${ }^{\$ 7}$. Dunque, tutto ciò che è umano, anzi tutto ciò che è vita o ancora, tutto ciò che riguarda il cosmo ci appartiene. Nulla ci è estraneo. E' l'essere la misura della coscienza, non nel senso che la trascende ma nel senso che la spinge a non chiudersi alle sue infinite manifestazioni, sempre oltre il traguardo raggiunto. L'essere va oltre ciò che appare e di cui siamo consapevoli ${ }^{88}$.

\section{E. CARATTERE FUNZIONALE DELLA RAZIONALITÀ ALL'OBLAZIONE}

In quest'ottica la razionalità appare del tutto funzionale alla presa d'atto della nostra responsabilità, in quanto creature con un respiro cosmico, poste al vertice del creato. Conseguente al recupero della progettualità divina del nostro essere, la razionalità assolve al compito di tradurre le nostre scelte in

\footnotetext{
${ }^{86}$ Givone, Metafisica della peste, 64. Perché nasce "difettivo", l'uomo diffonde il contagio, accrescendo la conflittualità. L'infezione si manifesta vivendo. Il non averne coscienza è la forma più deleteria di diffusione. Infatti, «quando il nulla ha eroso e consumato la possibilità stessa di distinguere fra bene e male, con la conseguenza di rendere indifferente la scelta, ogni scelta risulta perciò gratuita e affidata al caso, se non all'inclinazione o all'impulso del momento». Auschwitz: come è stato possibile? Poiché è accaduto, di chi la colpa? Di tutti. Singolarmente presi. Criminale è solo la persona singola. Tollerare i crimini, sostenendo il regime, assistere senza reagire, favorire la piega sociale che poi si rivelerà omicida. Se il capo di imputazione è comune a tutti i membri del corpo sociale - la superficialità e la condivisione di un nuovo stile di vita - imputabile è solo la persona singola.

${ }^{87}$ Edgar MORIN, La fraternità perché? Resistere alla crudeltà del mondo, trad. Nicola Manghi (Roma: Ave 2019), 55.

${ }^{88}$ Bonaventura, Quaestiones de scientia Christi, q. 4, resp, n. 27: «Cum duplex sit portio animae, superior et inferior, ratio inferior ortum habet a superiori, et non e converso». Ibid., n. 31 : «Anima nata est converti super intelligibile, quod est extra, et super intelligibile, quod est supra».
} 
gesti concreti, dotati di ampio respiro, e di misurare la gravità di ciò che accade, in vista del contributo adeguato alle attese dell'altro - singolo, gruppo o nazione. Il mondo è la realizzazione della volontà creativa di Dio; la Chiesa è l'istituzionalizzazione della sua volontà salvifica; la storia è il compendio dei progetti che singolarmente o comunitariamente gli uomini hanno elaborato; ognuno di noi è la realizzazione di specifici disegni di vita. L'unificazione di questi grandi capitoli ha luogo in misura che la ragione illumina il nostro coinvolgimento in Dio, nel mondo, nella storia, senza tirar fuori Dio dalla storia e dal mondo, la storia e il mondo da Dio, la Chiesa da Dio e dall'io, l'io dalla Chiesa, dalla storia e dal mondo. E' in questa interazione la conferma dell'indole cosmica del nostro essere e l'ambito della nostra responsabilità. La molla che attiva questo processo non è la razionalità, nel senso che la risposta a colui o coloro con cui si entra in rapporto dialogale è razionale, ma non è provocata dalla razionalità, ma dall'onda oblativa dell'essere. La razionalità resta insostituibile, non però originaria e determinante. Al primo posto la libertà, declinata secondo le più disparate modalità.

\section{CONCLUSIONE}

1. La figura del padre assunta a cifra del pensare francescano conferma che alla fonte dell'essere va posta la libertà, la cui luce aiuta a intendere che tutto ciò che è va letto secondo la logica del dono, sia per quanto concerne il mistero della Trinità e sia per quanto concerne la genesi del mondo. L'accentuazione francescana della libertà riguarda non solo l'atto creativo, ma anche il contenuto dell'atto creativo - Dio non è solo libero di creare, ma anche di creare questo e non quello, sicché una cosa è grande perché voluta, e non voluta perché grande. E' la volontà la padrona del territorio, non la ragione. Da qui l'originalità dell'atto creativo e insieme la gratuità di ciò che viene posto in essere. In questo contesto lo sguardo sul mondo è di segno ottimistico, sia in direzione trascendente che in direzione immanente. In direzione trascendente perché è opera di un Dio benevolo, che si esprime donando, imprimendo sulle creature $i$ segni di un linguaggio la cui decifrazione impegna a più livelli e in più direzioni, non in senso conflittuale o dispersivo ma in senso partecipativo e convergente. In direzione immanente in quanto in rapporto alla nostra storia evolutiva siamo invitati a porre l'accento sul fatto che siamo i discendenti di antenati di successo, 
i quali hanno dato prova di grande creatività sviluppando comportamenti flessibili verso gli stimoli ambientali. Se siamo qui vuol dire che siamo dotati di strumenti adatti ad affrontare situazioni difficili nonostante la nostra fragilità biologica. I pericoli esistono, ma disponiamo degli strumenti per gestirli, purché però messi a frutto in sintonia con il gruppo. La natura è grandiosa perché comunitaria ${ }^{89}$. La sua esplorazione può prendere molte direzioni - i saperi sono destinati a moltiplicarsi - nel rispetto però della sua indole originaria, e cioè che non è una realtà neutra, di nessuno e a nessuno affidata, ma è un giardino, affidato alla nostra cura perché sia la casa ospitale di tutti. La logica da mettere in campo non è di segno conflittuale o possessivo. Il che significa che il mondo e noi in esso siamo lo scrigno di Dio, da esplorare per ammirarne le meraviglie e agevolarne la manifestazione. Ebbene, sullo sfondo di questa ricostruzione si comprende che l'età moderna prende un'altra strada non perché si imbatta in una prospettiva autoritaria, ma perché ha davanti una prospettiva che ha a fondamento la volontà, non la ragione, l'essere come dono, non l'essere come diritto. Tutti gli altri motivi di dissenso o di critica, soprattutto di matrice politica, sono secondari, di sostegno, non sostanziali. La distanza tra l'età medievale, soprattutto nella lettura francescana, e l'età moderna è qualitativa.

2. La seconda tesi è che la traduzione di questa ontologia dell'essere come dono ha luogo grazie ad azioni non rivendicative o possessive, ma creative e oblative. E' lo scenario messo in evidenza dal volontarismo francescano, e cioè il mondo è il dono di Dio e la logica che presiede al suo divenire è la logica oblativa, nel senso che non fa che manifestare i suoi tesori con fenomeni spettacolari. Il mondo è grandioso. Altrettanto si dica dell'uomo. La sua avventura per quanto complessa è illuminata dalla presenza di Dio che non solo lo ha voluto, ma si è messo a sua disposizione, corroborando la logica oblativa. L'essere è oblazione. Unica la logica che illumina l'eterno e solca il tempo, la logica oblativa. A livello ontologico, l'essere non è, ma si dà; a livello antropologico il primato spetta alla volontà non alla ragione; a livello etico il metro valutativo non è ciò che si è o si ha ma ciò che si dà; a livello teologico, Dio non è ma si dona. Si tratta di una netta presa di distanza dalla logica competitiva e conflittuale della tradizione occidentale, propriamente possessiva, illuminata dalla logica della necessità, dall'ontologia dell'essere come diritto, dall'antropologia del primato della ragione, dall'etica del primato di ciò che si è e si ha.

${ }^{89}$ Daniel T. Blumstein, The Nature of Fear. Survival Lessons from the Wild (Cambridge, MA: Harvard University Press, 2020). 
3. La terza tesi che si è cercato di argomentare riguarda il presupposto della dimensione oblativa, costituito dalla responsabilità. La trama di ciò che mettiamo in essere, nell'ottica del primato della volontà, rappresenta lo spazio del senso dell'essere. Il contingente è ciò che accade, ma che può non accadere o accadere altrimenti ${ }^{90}$. E' l'area della libertà. Ebbene, proprio perché accade ma non necessariamente, il primato della volontà ci induce a sostenere che accade in quanto lo abbiamo voluto per vie dirette o per vie indirette. La trama degli eventi dal livello empirico viene portata al livello etico, in quanto cioè implica la domanda: e tu come hai contribuito al loro accadere? in quale misura rientri in quella trama? ${ }^{91}$ Se è vero che ognuno di noi è il compendio delle infinite circostanze della vita, un riassunto dell'avventura del cosmo, è conseguente che si parli di comunione a tutti i livelli, ed è stravagante il considerarsi fuori di questa logica che vede confluire in un'unica grande onda di vita ciò che si fa e si dice. Intesa in senso ampio, la cultura è il volto umano di quest'interazione, secondo modalità diverse in base ai ruoli che si coprono. Anche se ha un taglio poetico, pare sensato il dire che anche il battito d'ala di una farfalla è corresponsabile della tempesta nel Pacifico. E' a questa grande avventura del cosmo, della vita e dell'umanità il padre cerca di educare, sollecitando a guardare lontano, a sentire il peso e insieme l'emozione del nostro coinvolgimento. E' questo il motivo intorno a cui la teologia di Bonaventura ruota, e cioè il creato è una sorta di partecipazione alla processione eterna del Verbo dal Padre e questa è un'espressione della relazione di amore tra il Padre e il Figlio. Il pensare, il progettare e l'operare non devono aver luogo senza questo respiro cosmico, loro misura e criterio di valutazione ${ }^{92}$. Il che possiamo evocarlo richiamando la rappresentazione di Francesco con la croce dell'umanità sulle spalle, e cioè Francesco consapevole della lacerazione di mondi che non comunicano ma si fronteggiano e si scontrano. Francesco non

${ }^{90}$ Ord. I, d. 2, p. 1, q. 1-2, n. 86: «Non voco hic contingenter causatum quodcumque non necessarium vel non sempiternum, sed cuius oppositum posset fieri quando illud fit; ideo dixi 'contingetr causatur', et non 'aliquid est contingens'».

${ }^{91}$ Scotus, Questiones super libros Metaphysicorum Aristotelis IX, q. 15, n. 65: "Voluntas, quando est in aliqua volitione, tunc contingenter est in illa, et ita volitio tunc contingenter est ab ipsa; [...] Et sicut illa contingenter inest, ita voluntas tunc est potentia potens respectu oppositi".

${ }_{92}^{2}$ II Sent., d. 16, a. 1, q. 1: «Deus [...] cum sit summa potestas et maiestas, fecit omnia ad sui laudem, cum sit summa lux, fecit omnia ad sui manifestationem, cum sit summa bonitas, fecit omnia ad sui communicationem. Non est autem perfecta laus nisi adsit qui approbet, nec est perfecta manifestatio nisi adsit qui intelligat, nec perfecta communicatio bonorum nisi adsit qui eis uti valeat. Et quoniam laudem approbare, veritatem scire, dona in usum assumere non est nisi solummodo rationalis creaturae, ideo non habent ipsae creaturae irrationales immediate ad Deum ordinari, sed mediante creatura rationali». 
si ritiene fonte dei mali del mondo e tuttavia prende su di sé la croce di tutti i mali - la croce dell'umanità. Ecco la sua paternità. E' il controcanto al Cantico delle creature o anche è la solidarietà nella colpa. Aiutare il prossimo significa condividere ciò che accade, rispondendo a tutti di tutto, membri della stessa famiglia, tutti nella stessa barca. Responsabilità, solidarietà e fraternità: è la triade che ben compendia la densità teoretica della dimensione oblativa della volontà creativa e dunque le implicazioni le più significative del trascendimento del primato della coscienza prometeica o di autoaffermazione a favore dell'ascolto e della partecipazione, anima della paternità.

Dunque, più che privilegiare un determinato percorso, assumendo come esemplare la figura del padre si avanza l'idea che qualunque scelta filosofica debba essere sorretta da un'autentica anima oblativa. E' importante notarlo, il francescano non ritiene decisivo alcun indebolimento metafisico, compreso il percorso della secolarizzazione, perché entro la logica oblativa conta ciò che si dà, ma soprattutto il fatto che si dia, qualunque sia la forma e il contenuto, cioè, senza imporre, offrendo, mettendo a disposizione, con l'unico intento di ampliare gli spazi di vita e di pensiero. Non pare improduttivo tornare alla domanda classica - "Perché l'essere anziché il nulla?" - e portarsi oltre il nulla negativo di Leibniz, oltre il nulla enigmatico di Schelling, come oltre il nulla liberatorio delle filosofie deboliste, sostando entro il nulla assoluto della scuola francescana ${ }^{93}$, con 1'obiettivo di sottolineare che il padre ci ha tratti all'essere da tale nulla, grazie a un gesto che per un verso ribadisce la nostra autoinsufficienza e dunque la nostra dipendenza o passività, e per l'altro il carattere fondamentale della gratitudine. Il Nulla non può forse reincantarci, inducendoci a pensare che un tempo nulla era, e che poi un gesto di assoluta liberalità ha provocato questa esplosione di luce? La modernità, muovendo dal diritto-a-essere - le cose sono perché razionali e in base al grado di razionalità - ha lasciato in ombra e lentamente negato ciò che nell'ottica francescana è essenziale, e cioè la gratitudine. E' sulla consapevolezza del nulla originario che la gratitudine per ciò che si è e si ha diventa bisogno di esplorare e desiderio di capire. Al termine della stagione rivendicativa dei diritti soggettivi, sociali e individuali, credo che occorra riapprendere a ringraziare per il fatto e del fatto che si è. Non si tratta di un dovere tra i doveri, bensì dell'anima dell'essere.

\footnotetext{
${ }^{93}$ Si confronti la Parte I: Creatio ex nihilo del volume Discussioni sul nulla tra medioevo ed età moderna (Firenze: Leo S. Olschki, 2009), 3-119, in particolare il contributo di Paolo SINISCALCHI, "La creatio ex nihilo nel pensiero dei più antichi scrittori cristiani" (pp. 3-20) e di Massimiliano LENZI (ed.), Il nulla nelle Confessioni di Agostino tra creazione e conversione (pp. 21-36).
} 


\section{BIBLIOGRAFIA}

FONTI

\section{BONAVENTURA:}

Doctoris Seraphici S. Bonaventurae S. R. E. episcopi cardinalis opera omnia... (Commentaria in quatuor libros Sententiarum Magistri Petri Lombardi, Itinerarium mentis in Deum, De Mysterio Trinitatis, Lignum vitae, Quaestiones de scientia Christi, Collationes in Hexaëmeron, Breviloquium). 10 voll. Quaracchi, Firenze, 1882-1902.

Duns SCOTUS:

Cuestiones cuodlibetales. Edited by Felix Alluntis. Madrid: Bibliteca de Autores Christianos, 1968.

Joannis Duns Scoti Doctoris Subtilis, Ordinis Minorum Opera Omnia... (Lectura, Ordinatio, Reportata Parisiensia, Quaestiones Quodlibetales, Quaestiones super libros Metaphysicorum Aristotelis). A cura di Luis Vivès. Paris 1891-1895.

FRANCESCO D'ASSISI:

Scritti. A cura di Maria Pia Alberzoni. Padova: Editrici Francescane, 2002.

ALTRI:

Scripta Leonis, Rufini et Angeli Sociorum S. Francisci. Oxford Medieval Texts. A cura di Rosalind B. Brooke. Oxford: Clarendon Press, 1970.

ANONIMO DELla PoRZIUNCOLA. Speculum perfectionis status fratris Minoris. Edizione critica e studio storico-letterario a cura di Daniele Solvi. Firenze: Edizioni del Galluzzo, 2006.

\section{PubBlicAZIONI}

Arendt, Hanna. La vita della mente. Tradotto da Giorgio Zanetti, Bologna, Il Mulino, 1987.

BenedetTi, Marina. “'Ma qual è la vera letizia'. Realtà e metamorfosi di Francesco”. In Francesco da Assisi. Storia, arte, mito, a cura di Marina Benedetti e Tomaso Subini, 29-40. Roma: Carocci, 2019.

Blumstein, Daniel T. The Nature of Fear. Survival Lessons from the Wild. Cambridge, MA: Harvard University Press, 2020.

Brague, Rémi. Il futuro dell'Occidente. Tradotto da Adriana Soldati. Milano: Rusconi, 1998.

Cantarella, Eva. Come uccidere il padre. Genitori e figli da Roma a oggi. Milano: Feltrinelli, 2020.

CAvaciuti, Santino. Libertà e trascendenza. Soveria Mannelli: Rubbettino, 2018.

DE DHAEM, Armaury Begasse, "Scriptura sive theologia o come Dio viene all'uomo. Il proprium di San Bonaventura secondo Étienne Gilson ed Emmanuel Falque”. In Trilogia bonaventuriana, a cura di Carmelo Pandolfi e Rafael Pascual, 639-84. Roma: IF Press, 2020.

FALQUe, Emmanuel. Saint Bonaventure et l'entrée de Dieu en théologie. Paris: Vrin, 2000.

ForTHOMME, Bernard. Il canto del corpo ardente. La stimmatizzazione di san Francesco di Assisi. Padova: Messaggero, 2012.

Frugoni, Chiara. Un presepio con molte sorprese. San Francesco e il Natale di Greccio. Roma: Mauvais Livres, 2020. 
Givone, Sergio. Metafisica della peste. Colpa e destino. Torino: Einaudi, 2012.

Gregory, Tullio. Mundana sapientia. Forme di conoscenza nella cultura medievale. Roma: Edizioni di Storia e Letteratura, 1992.

HeIDEGGER, Martin. I problemi fondamentali della fenomenologia. Genova: Il Melangolo, 1988.

HeIDEGGER, Martin. Introduzione alla metafisica. Milano: Mursia, 1986.

HEIDEGger, Martin. La questione della cosa. La dottrina kantiana dei principi trascendentali. Napoli: Guida, 1989.

LENZI, Massimiliano. "Il nulla nelle Confessioni di Agostino tra creazione e conversione". In Discussioni sul nulla tra medioevo ed età moderna, a cura di Massimiliano Lenzi e Alfonso Maierù, 21-36. Firenze: Olschki, 2009.

Le Goff, Jacques. San Francesco d'Assisi. Tradotto da Lisa Baruffi e Amedeo De Vincentiis. Bari: Laterza, 2002.

Malaguti, Maurizio. La metafisica del volto. Una lettura di Dante. Milano: Edizioni Biblioteca Francescana, 2020.

Merton, Thomas. Gli abissi infiniti del cielo. Tradotto da Chiara Benedetti. Brescia: Queriniana, 2019.

MORIN, Edgar. La fraternità perché? Resistere alla crudeltà del mondo. Tradotto da Nicola Manghi, Roma: Ave, 2019.

PACIOCCO, Roberto. "'Sub iugo servitutis'. Francesco, i Francescani e la 'militia Christi”". In "Militia Christi" e Crociata nei secoli XI-XIII. Atti della undecima Settimana internazionale di studio, Mendola 28 agosto - 1 settembre 1989, 699-715. Milano: VeP, 1992.

SinisCALCHI, Paolo. "La creatio ex nihilo nel pensiero dei più antichi scrittori cristiani". In Discussioni sul nulla tra medioevo ed età moderna, a cura di Massimiliano Lenzi e Alfonso Maierù, 3-20. Firenze: Olschki, 2009.

Strummiello, Giuseppina. Il logos violato. La violenza nella filosofia. Bari: Dedalo 1991.

Thompson, Augustine. Francesco d'Assisi una nuova biografia. Bari: Edizioni di Pagina, 2016.

Tresmontant, Claude. La Christologie du B. Jean Duns Scot, l'Immaculée Conception et l'avenir dell'Eglise. Note complementaire à propos du peché originel. Paris: François-Xavier de Guibert, 1996.

VAtтimo, Gianni. La vita dell'altro. Bioetica senza metafisica. Napoli: Marco Costantino, 2006.

\section{RECUPERO DELLA FIGURA DEL PADRE AI MARGINI DEL MEDIOEVO FRANCESCANO}

\section{So m m a rio}

Tre le tesi che si è cercato di argomentare sullo sfondo dell'assunto generale, secondo cui l'età moderna prende le distanze dall'età medievale in genere e francescana in particolare non perché autoritaria - il figlio che si ribella al padre secondo l'abituale cliché storiografico - ma perché vuole impiantare la lettura dell'essere non più sulla volontà di Dio e dell'uomo, ma su parametri ispirati al primato della ragione, autonoma e autosufficiente, tribunale inappellabile della verità. Sullo sfondo di quest'assunto, la prima tesi che si cerca di argomentare è che il "padre", in quanto cifra di volontà creativa, sembra che sia la figura più rappresentativa del "pensare francescano"; la seconda è che il padre traduce tale volontà non prendendo, ma dando, 
non dominando ma servendo; la terza è che il padre incarna in maniera singolare la responsabilità, non circoscritta alla propria individualità, ma aperta all'altro - famiglia, gruppo, nazione, mondo. In breve, il francescano pare che guardi al padre come all'espressione più compiuta della sua prospettiva filosofico-teologica, in quanto rappresenta colui che dona tutto ciò che è e che ha, con responsabilità, in linea con quel volontarismo per il quale le creature sono non perché avessero un qualche diritto a essere, ma perché volute in radicale gratuità.

Parole chiave: padre; liberta; cultura Francesco d'Asisi; oblazione.

\section{ODZYSKANIE OBRAZU OJCA NA MARGINESIE ŚREDNIOWIECZA FRANCISZKAŃSKIEGO}

$$
\text { Streszczenie }
$$

Staraliśmy się omówić trzy argumenty odnoszące się do tezy, że epoka współczesna dystansuje się od epoki średniowiecznej posiadającej charakter franciszkański nie dlatego, że jest autorytarna (według stereotypu historiograficznego przedstawiającego syna, który buntuje się przeciwko ojcu), ale ponieważ chce zaszczepić odczytywanie bycia nie w woli Boga i człowieka, ale $\mathrm{w}$ parametrach inspirowanych prymatem autonomicznego i samowystarczalnego rozumu, będącego bezapelacyjnym trybunałem prawdy. W tezie pierwszej argumentujemy, że "ojciec", będący w jakiejś mierze wolą kreatywną jest obrazem reprezentatywnym dla myśli franciszkańskiej. W tezie drugiej sugerujemy, że "ojciec" przekłada powyższą wolę nie biorąc, ale dając, nie dominując, ale służąc. W trzeciej tezie pokazujemy, że "ojciec" w wyjątkowy sposób wciela odpowiedzialność przypisaną do własnej indywidualności, ale jednocześnie otwartą na drugiego na rodzinę, grupę, naród, świat. Wydaje się, że franciszkanin patrzy na ojca jako na wyrażenie odnajdującej swoje spełnienie w jego własnej perspektywie fílozoficzno-teologicznej, w której reprezentuje on kogoś, kto z pełną odpowiedzialnością daje wszystko, czym jest i co posiada. W perspektywie tej odpowiedzialności woluntaryzm jawi się jako postawa, dzięki której stworzenia istnieją nie dlatego, że posiadają prawo do istnienia, ale dlatego, że są chciane $\mathrm{i}$ to $\mathrm{z}$ radykalną bezinteresownością.

Słowa kluczowe: ojciec; wolność; kultura; Franciszek z Asyżu; ofiara.

\section{RECOVERY OF THE FATHER FIGURE AT THE MARGINS OF THE FRANCISCAN MIDDLE AGES}

\section{Su m m a ry}

There are three arguments that we have tried to discuss with respect to the general assumption that the modern age distances itself from the medieval age in general and the Franciscan age in particular, not because it is authoritarian (according to the typical historiographic cliche, which depicts a son who rebels against his father) but because it wants to embed the interpretation of being no longer in the will of God and man, but in parameters inspired by the primacy of reason, autonomous and self-sufficient - the irrefutable tribunal of truth. It is argumented in the first thesis that the "father", as a figure of creative will to some extent, seems to be the most representative figure of "Franciscan thinking". The second thesis is that the father fulfils this will 
not by taking, but by giving, not by dominating but by serving. The third thesis is that the father incarnates in an extraordinary way the responsibility, ascribed to his own individuality, but at the same time open to others: family, group, nation, world. In short, the Franciscan seems to look at the father as the most complete expression of his philosophical-theological perspective, in that he represents the one who gives all that he is and has, with full responsibility. Given that responsibility, voluntarism appears as an attitude in which creatures exist not because they have a right to be, but because they are wanted with radical disinterestedness.

Keywords: father; freedom; culture; Francis of Assisi; sacrifice. 